This item was submitted to Loughborough's Research Repository by the author.

Items in Figshare are protected by copyright, with all rights reserved, unless otherwise indicated.

\title{
Mixed mode partition theories for one dimensional fractures
}

PLEASE CITE THE PUBLISHED VERSION

http://dx.doi.org/10.1016/j.engfracmech.2011.11.013

PUBLISHER

(c) Elsevier

VERSION

AM (Accepted Manuscript)

LICENCE

CC BY-NC-ND 4.0

REPOSITORY RECORD

Wang, Simon, and Christopher M. Harvey. 2011. "Mixed Mode Partition Theories for One Dimensional Fractures". figshare. https://hdl.handle.net/2134/9168. 
This item was submitted to Loughborough's Institutional Repository (https://dspace.lboro.ac.uk/) by the author and is made available under the following Creative Commons Licence conditions.

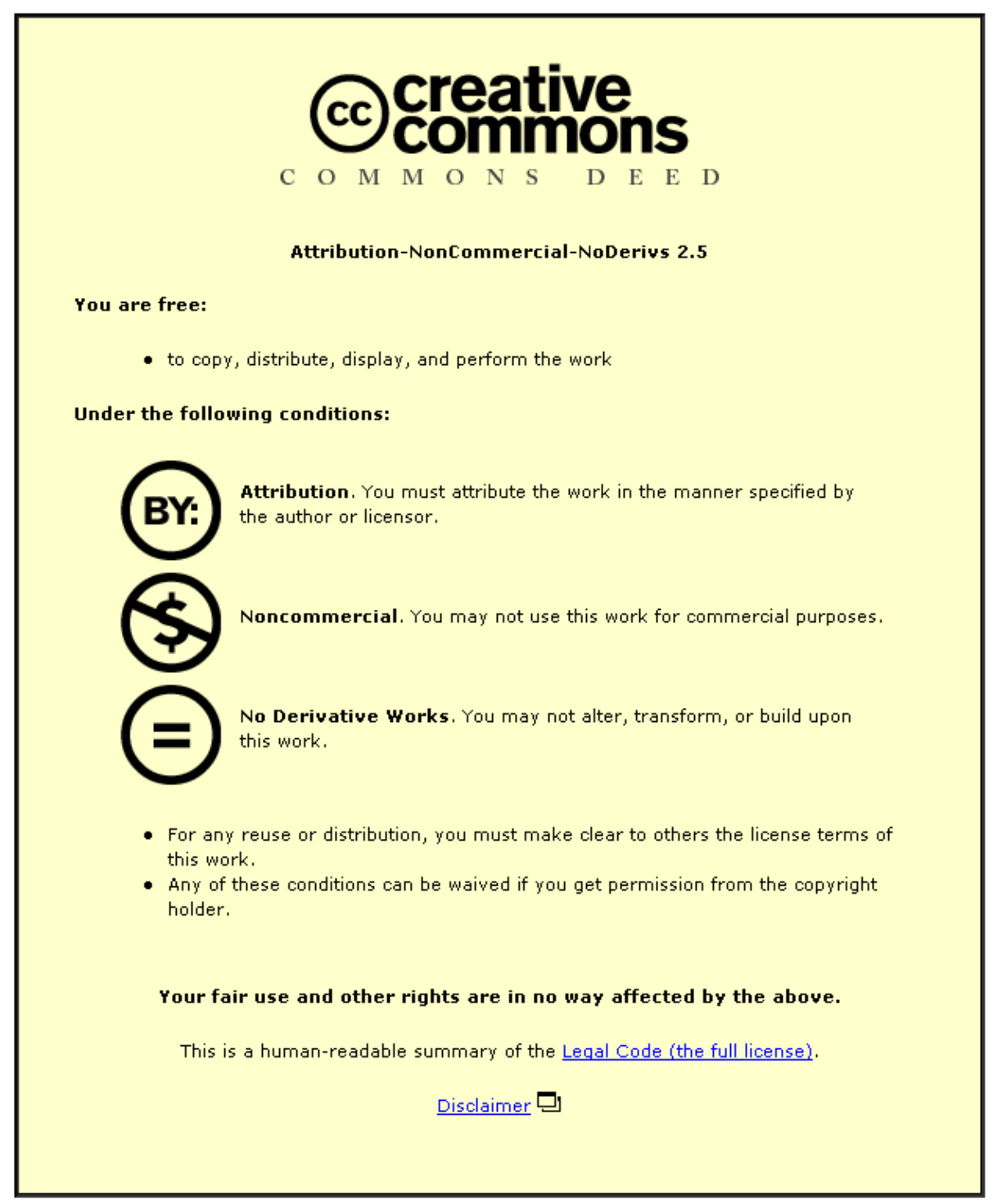

For the full text of this licence, please go to: http://creativecommons.org/licenses/by-nc-nd/2.5/ 


\title{
Mixed mode partition theories for one dimensional fracture
}

\author{
S. Wang ${ }^{\mathrm{a}, *}$ and C. Harvey ${ }^{\mathrm{a}}$ \\ ${ }^{a}$ Department of Aeronautical and Automotive Engineering, Loughborough University, Loughborough, \\ Leicestershire, LE11 3TU, United Kingdom
}

\begin{abstract}
The crack in a double cantilever beam is the most fundamental one-dimensional fracture problem. It has caused considerable confusions due to its in-depth subtleness and complex entanglement with different theories and numerical simulations. The present paper presents completely analytical theories based on Euler and Timoshenko beam theories using a brand new approach which reveals the hidden mechanics of the problem. Orthogonal pairs of pure modes are found and used to partition mixed modes. The developed theories are extensively validated against numerical simulations using finite element methods. Moreover, the fracture mode partition space is thoroughly investigated and crack tip running contact is found which results in a region of pure mode II. The theories are finally applied to general one-dimensional fracture in beams and axisymmetric plates.
\end{abstract}

Keywords: Energy release rate, Orthogonal pure fracture modes, Mixed mode partitions

\section{Introduction}

Double cantilever beams (DCBs) are typical representatives of one-dimensional fracture problems and often used to determine critical energy release rates of materials. Fracture mode partitions play a key role in the development of crack propagation criteria. The Williams partition rules were given in his pioneering work [1]. Another piece of pioneering work [2] was given by Schapery and Davidson who claimed that Euler beam theory does not provide quite enough information to obtain a decomposition of energy release rate into opening and shearing mode components. Hutchinson and Suo [3] presented their combined numerical and analytical rules based on stress intensity factors and claimed Williams rules [1] containing conceptual errors. Some other earlier works are given in references $[4,5,6]$. Several recent research works on the topic are quoted here among many others. They are Wang and Qiao [7], Nguyen and Levy [8], Yan and Shang [9], Ouyang and Li [10], and Zou et al. [11, 12]. As far as the authors' knowledge is concerned, the previous works have not reported the solutions presented in this work. On the computational front, Hong and Shim [13] reported that Williams rules [1] disagreed with their finite element method (FEM) simulations with off centre cracks. Zhang [14] also noted that in his work. In this paper, completely analytical partition theories are developed based on both Euler and Timoshenko beam theories by using a completely new approach. The work reveals the indepth mechanics of the problem and complex entanglements between numerical simulations and different analytical theories. It aims to clarify all the confusions in both numerical and analytical aspects. Specific comments on earlier reference works, particularly $[1,2,3]$ will be given at appropriate points in the development of the paper for the sake of easy description and

\footnotetext{
* Corresponding Author

Email addresses: s.wang@lboro.ac.uk (S. Wang), c.m.harvey@lboro.ac.uk (C. Harvey)
} 
understanding. Various parts of the present work have been reported on several occasions by the authors [15-21]. The structure of the paper is as follows. The theories are given in Section 2. Numerical tests are presented in Section 3. Conclusions are made in Section 4.

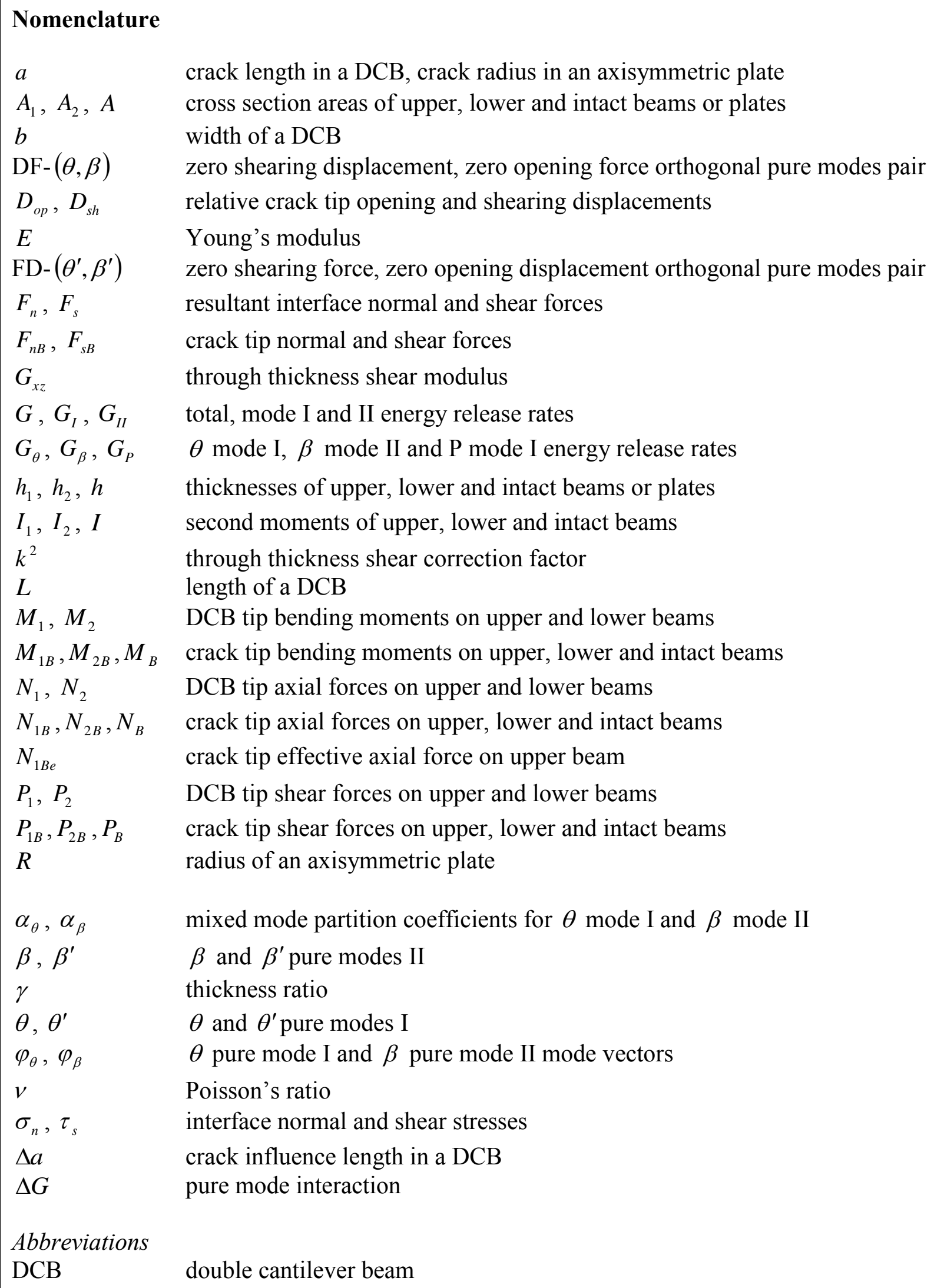




\section{Theories}

\subsection{Orthogonal fracture mode partition}

Fig. 1 shows a DCB with its geometry and two tip bending moments. No shear and axial tip loads are applied at this stage for simplicity and in order to focus on important mechanical understanding. Assuming no interface contact within the cracked region, the strain energy of the DCB can be written as in Eq. (1) for either an Euler or a Timoshenko beam of linear elastic materials.

$$
U=\frac{1}{2 E}\left[\int_{0}^{a}\left(\frac{M_{1}^{2}(x)}{I_{1}}+\frac{M_{2}^{2}(x)}{I_{2}}\right) d x+\int_{a}^{L} \frac{M^{2}(x)}{I} d x\right]
$$

The origin of $x$ coordinate is at DCB tip and towards to the left in Eq. (1). The energy release rate $G$ at crack tip point B is therefore obtained as

$$
G=\frac{1}{b} \frac{\partial U}{\partial a}=\frac{1}{2 b E}\left(\frac{M_{1 B}^{2}}{I_{1}}+\frac{M_{2 B}^{2}}{I_{2}}-\frac{M_{B}^{2}}{I}\right)
$$

Here, $b$ is the width of the beam and the other quantities have their conventional meanings. It is seen that $G$ is a local quantity depending on the quantities at the crack tip cross-section, i.e. $M_{1 B}, M_{2 B}$ and $M_{B}$. Also, $G$ is of quadratic form that is positive definite and can be expressed in orthogonal forms. To do so, $M_{1 B}$ and $M_{2 B}$ are expressed as

$$
\left\{\begin{array}{l}
M_{1 B} \\
M_{2 B}
\end{array}\right\}=\alpha_{\theta}\left\{\begin{array}{l}
1 \\
\theta
\end{array}\right\}+\alpha_{\beta}\left\{\begin{array}{l}
1 \\
\beta
\end{array}\right\}=\alpha_{\theta} \varphi_{\theta}+\alpha_{\beta} \varphi_{\beta}
$$

When either $\theta$ or $\beta$ is given, the other can be found using the orthogonal condition with respect to the coefficient matrix of the form. Therefore, a pair of orthogonal modes, i.e. $\varphi_{\theta}$ and $\varphi_{\beta}$ can be found. The quantities $\alpha_{\theta}$ and $\alpha_{\beta}$ are mode partition coefficients and can be determined from Eq. (3) for any given $M_{1 B}$ and $M_{2 B}$. Then, substituting Eq. (3) into Eq. (2) yields

$$
G=\frac{1}{2 b E}\left[\alpha_{\theta}^{2}\left(\frac{1}{I_{1}}+\frac{\theta^{2}}{I_{2}}-\frac{(1+\theta)^{2}}{I}\right)+\alpha_{\beta}^{2}\left(\frac{1}{I_{1}}+\frac{\beta^{2}}{I_{2}}-\frac{(1+\beta)^{2}}{I}\right)\right]=\alpha_{\theta}^{2} G_{\theta}+\alpha_{\beta}^{2} G_{\beta}
$$

where $G_{\theta}$ and $G_{\beta}$ are the energy release rates corresponding to the orthogonal modes $\varphi_{\theta}$ and $\varphi_{\beta}$, respectively. In general, they are not the respective pure opening mode I and pure shearing mode II as an infinite number of orthogonal modes exist. Consequently, it is natural to ask whether specific values of the pair $(\theta, \beta)$ exist which give pure modes I and II, respectively. A further enquiry is that when the pair $(\theta, \beta)$ is found, does the Eq. (4) represent the true energy release rate partition? The following study concerns these questions. After these questions are answered, the theory is extended to study the general case where axial and shear forces are included. 


\subsection{Two orthogonal pairs of locally pure modes}

From mechanical considerations two pairs of locally pure modes are expected. The first pair corresponds to zero crack tip relative shearing displacement, i.e. mode I, and zero crack tip opening force, i.e. mode II. The second pair corresponds to zero crack tip shearing force, i.e. mode I, and zero crack tip relative opening displacement, i.e. mode II. They are designated as the $D F-(\theta, \beta)$ pair and the $F D-\left(\theta^{\prime}, \beta^{\prime}\right)$ pair, respectively. The $D$ and $F$ indicate that the corresponding pure modes are due to zero crack tip relative displacements and zero crack tip forces, respectively. These two pairs of pure modes are determined in this section.

To begin with, it is assumed that the mechanical influence of the crack extends to a point $\mathrm{A}$, $\Delta a$ distance ahead of the crack tip B as shown in Fig. 2a. It is noted that Fig. $2 \mathrm{~b}$ only shows the sign convention of the interface normal stress $\sigma_{n}$ and shear stress $\tau_{s}$ instead of any representative distribution of them. By using curvature continuity conditions at point $\mathrm{A}$, the resultant shear force $F_{s}$ due to $\tau_{s}$ and the resultant moment $M_{n}$ due to $\sigma_{n}$ can be obtained as [15-21]

$$
F_{s}=\int_{0}^{\Delta a} \tau_{s} d x=\frac{6 \gamma}{h_{1}(1+\gamma)^{3}}\left(M_{1 B}+M_{2 B}\right)
$$

and

$$
M_{n}=b \int_{0}^{\Delta a} \int_{0}^{x} \sigma_{n} d x d x=b \int_{0}^{\Delta a} \sigma_{n}(\Delta a-x) d x=M_{n m}+F_{n} \Delta a
$$

where $M_{n m}$ is given by

$$
M_{n m}=-b \int_{0}^{\Delta a} x \sigma_{n} d x=\frac{1+3 \gamma}{(1+\gamma)^{3}}\left(\beta M_{1 B}-M_{2 B}\right)
$$

and the resultant normal force $F_{n}$ due to $\sigma_{n}$ is zero since there are no crack tip shear forces. Eqs. (5) and (6) remain the same in both Euler and Timoshenko beam theories. Here, $\gamma=h_{2} / h_{1}$ is the thickness ratio, $\beta=\gamma^{2}(3+\gamma) /(1+3 \gamma)$ and the origin of the $x$ coordinate is at crack tip B and towards to the left.

A globally pure mode $\mathrm{I}$ is defined as $F_{s}=0$ leading to $M_{1 B}=-M_{2 B}$ which is written in Eq. (7) in the form of a mode vector.

$$
\varphi_{\theta_{E}^{\prime}}=\left\{\begin{array}{c}
1 \\
\theta_{E}^{\prime}
\end{array}\right\}=\left\{\begin{array}{c}
1 \\
-1
\end{array}\right\}
$$

Its orthogonal globally pure mode II, which is given by Eq. (8), can be found by using the orthogonal condition with respect to the coefficient matrix of the quadratic form of energy release rate as described in Section 2.1.

$$
\varphi_{\beta_{E}^{\prime}}=\left\{\begin{array}{c}
1 \\
\beta_{E}^{\prime}
\end{array}\right\}=\left\{\begin{array}{c}
1 \\
\gamma^{3}
\end{array}\right\}
$$

Physically, the orthogonal property means that the interaction between the two modes results in zero net amount of energy release rate. Apparently, this pair of globally pure modes happens to be Williams' modes [1] and is designated as the $F D-\left(\theta_{E}^{\prime}, \beta_{E}^{\prime}\right)$ pair here.

Similarly, a globally pure mode II is defined as $M_{n}=0$ leading to the following mode vector. 


$$
\varphi_{\beta}=\left\{\begin{array}{l}
1 \\
\beta
\end{array}\right\}=\left\{\begin{array}{c}
1 \\
\gamma^{2}(3+\gamma) /(1+3 \gamma)
\end{array}\right\}
$$

Its orthogonal globally pure mode I can be found as

$$
\varphi_{\theta}=\left\{\begin{array}{c}
1 \\
\theta
\end{array}\right\}=\left\{\begin{array}{c}
1 \\
-\gamma^{2}
\end{array}\right\}
$$

Notice that $\theta\left(\gamma^{-1}\right)=\theta^{-1}(\gamma)$ and $\beta\left(\gamma^{-1}\right)=\beta^{-1}(\gamma)$ due to mechanical symmetry. This pair is the Wang-Harvey pair [15-21] and designated as the $D F-(\theta, \beta)$ pair. In the following, the aim is to show that the $F D-\left(\theta_{E}^{\prime}, \beta_{E}^{\prime}\right)$ pair is only locally pure in Euler beam theory whilst the $D F-(\theta, \beta)$ pair is locally pure in both Euler and Timoshenko beam theories. Note that the local pureness is defined with respect to the crack tip B whilst the global pureness is defined with respect to the $\Delta a$ region as shown in Fig. 2.

By using Fig. 2 and Timoshenko beam theory, the relative opening displacement of beams 1 and 2 at the two points of $d a$ distance behind the crack tip are calculated in Eq. (11).

$$
D_{o p}=\frac{1}{2 E}\left(\frac{M_{1 B}}{I_{1}}-\frac{M_{2 B}}{I_{2}}\right) d a^{2}+\left(\psi_{1 B}-\psi_{2 B}\right) d a+\left(w_{1 B}-w_{2 B}\right)
$$

Note that the origin of coordinate $x$ in Eq. (11) is at the crack tip B and towards to the right, deflection $w$ is upwards and rotation $\psi$ is anticlockwise. For a rigid interface, $w_{1 B}-w_{2 B}=0$. The rotation term in Eq. (11) can be found in what follows. Within the $\Delta a$ region, the through thickness shearing equations are

$$
k^{2} G_{x z} A_{1}\left(w_{1}^{(1)}-\psi_{1}\right)=b \int_{0}^{x} \sigma_{n} d x
$$

and

$$
k^{2} G_{x z} A_{2}\left(w_{2}^{(1)}-\psi_{2}\right)=-b \int_{0}^{x} \sigma_{n} d x
$$

for beams 1 and 2, respectively, where $w^{(1)}=d w / d x, A$ represents cross section area, $G_{x z}$ is the through thickness shear modulus and $k^{2}$ is the shear correction factor, usually taken to be $5 / 6$ for isotropic materials. Note that the origin of coordinate $x$ in Eqs. (12) and (13) is at crack tip B and towards to the left and the rotation $\psi$ is clockwise. Due to the opening stress singularity at the crack tip B, the two through thickness shear strains are not continuous across the crack tip. Since both two rotations $\psi_{1}$ and $\psi_{2}$ are continuous although they are different, $w_{1}^{(1)}$ and $w_{2}^{(1)}$ are discontinuous at the crack tip and different behind the crack tip. However, it can be assumed that $w_{1}^{(1)}(d a)=w_{2}^{(1)}(d a)$ for a rigid interface which are the two mid-plane rotations of beams 1 and 2, respectively, , at $d a$ distance ahead of the crack tip B. Therefore, from Eqs. (12) and (13), the quantities $w_{1}^{(1)}(d a)$ and $w_{2}^{(1)}(d a)$ are obtained as

$$
w_{1}^{(1)}(d a)=\psi_{1}(d a)+\frac{F_{n B}}{k^{2} G_{x z} A_{1}}
$$

and

$$
w_{2}^{(1)}(d a)=\psi_{2}(d a)-\frac{F_{n B}}{k^{2} G_{x z} A_{2}}
$$


where $F_{n B}=b \int_{0}^{d a} \sigma_{n} d x$ is the crack tip opening force. Since $w_{1}^{(1)}(d a)=w_{2}^{(1)}(d a), \psi_{1}(d a)=\psi_{1 B}$ and $\psi_{2}(d a)=\psi_{2 B}$, Eqs. (14) and (15) give

$$
\psi_{1 B}-\psi_{2 B}=-\frac{F_{n B}}{k^{2} G_{x z}}\left(\frac{1}{A_{1}}+\frac{1}{A_{2}}\right)
$$

It is seen that the two crack tip rotations are generally different in Timoshenko beam theory. When shear modulus $G_{x z}$ tends to be infinite leading to Euler beam theory, they become equal. Substituting Eq. (16) into Eq. (11) yields

$$
D_{o p}=\frac{1}{2 E}\left(\frac{M_{1 B}}{I_{1}}-\frac{M_{2 B}}{I_{2}}\right) d a^{2}+\frac{F_{n B}}{k^{2} G_{x z}}\left(\frac{1}{A_{1}}+\frac{1}{A_{2}}\right) d a
$$

Note that a sign reversal has been done when substituting Eq. (16) into Eq. (11) as the rotation direction in Eq. (16) is opposite to that in Eq. (11). When using Euler beam theory, i.e. $G_{x z}=\infty$, the zero condition $D_{o p}=0$ gives the pure mode II in Eq. (8), i.e. the $\varphi_{\beta_{E}^{\prime}}$ mode. This proves that $\varphi_{\beta_{E}^{\prime}}$ mode is also locally pure in Euler beam theory. Therefore, its orthogonal mode $\varphi_{\theta_{E}^{\prime}}$ given in Eq. (7) is also a locally pure mode in Euler beam theory corresponding to zero crack tip shearing force, i.e. $F_{s B}=0$. When using Timoshenko beam theory for a finite value of $G_{x z}$, the zero condition $D_{o p}=0$ yields $F_{n B}=0$ instead of the $\varphi_{\beta_{E}^{\prime}}$ mode. Therefore, it can be concluded that the FD- $\left(\theta_{E}^{\prime}, \beta_{E}^{\prime}\right)=\left(-1, \gamma^{3}\right)$ pair is not a locally pure pair in Timoshenko beam theory for shear modulus of finite values. The simultaneous zero conditions $D_{o p}=0$ and $F_{n B}=0$ state that the two locally pure mode II modes coincide with each other corresponding to zero crack tip opening displacement and zero crack tip opening force. Effectively, there is only one pair of pure modes in Timoshenko beam theory. It will be shown shortly that it is the $D F-(\theta, \beta)$ pair.

By using Fig. 2, the relative axial displacement of the two points on the broken interfaces at $d a$ distance behind the crack tip B is calculated as in Eq. (18).

$$
D_{s h}=\left(u_{1 B}+\frac{h_{1}}{2} \psi_{1 d a}\right)-\left(u_{2 B}-\frac{h_{2}}{2} \psi_{2 d a}\right)
$$

where

$$
\psi_{1,2 d a}=\frac{M_{1,2 B} d a}{E I_{1,2}}+\psi_{1,2 B}
$$

Due to the following axial continuity condition

$$
u_{1 B}+\frac{h_{1}}{2} \psi_{1 B}=u_{2 B}-\frac{h_{2}}{2} \psi_{2 B}
$$

Eq. (18) becomes

$$
D_{s h}=\frac{1}{2 E}\left(\frac{h_{1} M_{1 B}}{I_{1}}+\frac{h_{2} M_{2 B}}{I_{2}}\right) d a
$$

Again, note that the origin of coordinate $x$ in Eqs. (18-21) is at the crack tip B and towards to the right and the mid-plane axial displacement $u$ is towards to the right as well. Also, note that Eq. (21) remains the same in both Euler and Timoshenko beam theories. The zero crack tip 
shearing displacement, i.e. $D_{s h}=0$ gives the pure mode I, i.e. the $\varphi_{\theta}$ mode in Eq. (10). This proves that the $\varphi_{\theta}$ mode is locally pure in both Euler and Timoshenko beam theories. Therefore, its orthogonal mode $\varphi_{\beta}$ given in Eq. (9) is also locally pure in both Euler and Timoshenko beam theories. It corresponds to zero crack tip opening force, i.e. $F_{n B}=0$. Since $F_{n B}=0$ leads to $D_{o p}=0$ as seen earlier, $D_{s h}=0$ will lead to zero crack tip shear force, i.e. $F_{s B}=0$ in Timoshenko beam theory. Therefore, the two pairs of locally pure modes in Timoshenko beam theory coincide with each other. That is, $D F-(\theta, \beta)=F D-\left(\theta_{T}^{\prime}, \beta_{T}^{\prime}\right)$.

More comments on the $D F-(\theta, \beta)$ pair are desirable.

- It can be shown that the normal interface stress $\sigma_{n}$ in the $\varphi_{\beta}$ mode is uniformly zero [1521] within the $\Delta a$ region including the crack tip point B. Obviously, in this situation, the two beams within the $\Delta a$ region deform like an intact whole beam except that a shear stress singularity exists at the very crack tip point $\mathrm{B}$, which results in a pure shear mode.

- The two crack tip rotations in the $\varphi_{\beta}$ mode are always equal to each other in Timoshenko beam theory.

- In Timoshenko beam theory, the $\varphi_{\beta}$ mode corresponding to $F_{n B}=0$ and the $\varphi_{\beta_{T}^{\prime}}$ mode corresponding to $D_{o p}=0$ coincide with each other. However, it is seen from Eq. (17) that the coincidence, i.e. $\beta_{T}^{\prime}$ approaching to $\beta$, is from above and is an asymptotic process. Extra care has to be taken in FEM simulation. Very fine meshes are required to ensure that $\beta_{T}^{\prime}$ converges to $\beta$. Otherwise, a $\varphi_{\beta^{\prime}}$ mode can be generated numerically with $\beta=\beta_{T}^{\prime}<\beta^{\prime}<\beta_{E}^{\prime}=\gamma^{3}$. Similarly, a $\varphi_{\theta^{\prime}}$ mode can also be generated numerically with $\theta=\theta_{T}^{\prime}<\theta^{\prime}<\theta_{E}^{\prime}=-1$.

- Since $D_{o p}<0$ beyond $\beta_{T}^{\prime}=\beta$ in Timoshenko beam theory, crack tip running contact occurs leading to a pure mode II region between $\beta_{T}^{\prime}$ and $\beta_{E}^{\prime}$.

In summary, in Timoshenko beam theory, $D F-(\theta, \beta)$ and $F D-\left(\theta_{T}^{\prime}, \beta_{T}^{\prime}\right)$ are the two pairs of locally pure modes. However, only $D F-(\theta, \beta)$ pair is globally pure as well. The second globally pure pair is $F D-\left(\theta_{E}^{\prime}, \beta_{E}^{\prime}\right)$. In Euler beam theory, $D F-(\theta, \beta)$ and $F D-\left(\theta_{E}^{\prime}, \beta_{E}^{\prime}\right)$ are the two pairs of locally pure modes which are also globally pure. It is seen that locally pure modes are also globally pure but it is not true vice versa.

\subsection{Mixed mode partitions using the $D F-(\theta, \beta)$ pair}

Interface stresses $\tau_{s}$ and $\sigma_{n}$ as shown in Fig. 2 are considered first. Eq. (22) gives the continuity condition of axial displacement at the interfaces of the two beams within the $\Delta a$ region.

$$
u_{1}+\frac{h_{1}}{2} \psi_{1}=u_{2}-\frac{h_{2}}{2} \psi_{2}
$$

where

$$
u_{1,2}=u_{1,2 B} \mp \frac{b}{E A_{1,2}} \iint \tau_{s} d x d x
$$

From Eq. (22), the shear stress $\tau_{s}$ is found as 


$$
\tau_{s}=\delta_{B}+\frac{3(1-\gamma)}{2 h_{1} \gamma} \int_{0}^{x} \sigma_{n} d x
$$

where $\delta_{B}$ is the shear stress singularity at the crack tip B arising from the $\varphi_{\beta}$ mode II and the second term is due to the normal stress $\sigma_{n}$ arising from the $\varphi_{\theta}$ mode I. Eq. (24) remains valid in both Euler and Timoshenko beam theories. The relationship between the crack tip shear force and opening force in the $\varphi_{\theta}$ mode I can be obtained can be obtained from Eq. (24).

$$
F_{s B \theta}=\frac{3(1-\gamma) F_{n B \theta} d a}{4 h_{1} \gamma}
$$

where $F_{s B \theta}=b \int_{0}^{d a} \tau_{s \theta} d x$ and $F_{n B \theta}=b \int_{0}^{d a} \sigma_{n \theta} d x$.

Now, mixed mode partitions are considered. For any given $M_{1 B}$ and $M_{2 B}$, the mode partition coefficients $\alpha_{\theta}$ and $\alpha_{\beta}$ in Eq. (3) are obtained in Eq. (26).

$$
\left\{\begin{array}{l}
\alpha_{\theta} \\
\alpha_{\beta}
\end{array}\right\}=\left[\begin{array}{ll}
\varphi_{\theta} & \varphi_{\beta}
\end{array}\right]^{-1}\left\{\begin{array}{l}
M_{1 B} \\
M_{2 B}
\end{array}\right\}=\frac{1}{\beta-\theta}\left\{\begin{array}{c}
\beta M_{1 B}-M_{2 B} \\
-\theta M_{1 B}+M_{2 B}
\end{array}\right\}
$$

The energy release rates $G_{\theta}$ and $G_{\beta}$ in Eq. (4) are calculated as

$$
G_{\theta}=\frac{24 \gamma}{E b^{2} h_{1}^{3}(1+\gamma)}
$$

and

$$
G_{\beta}=\frac{6}{E b^{2} h_{1}^{3}}\left[1+\frac{\beta^{2}}{\gamma^{3}}-\frac{(1+\beta)^{2}}{(1+\gamma)^{3}}\right]
$$

The total energy release rate is therefore given by

$$
G=\alpha_{\theta}^{2} G_{\theta}+\alpha_{\beta}^{2} G_{\beta}
$$

The partition of the total $G$ into $G_{I}$ and $G_{I I}$ is considered first within the context of Timoshenko beam theory. In Timoshenko beam theory, the $D F-(\theta, \beta)$ and $F D-\left(\theta_{T}^{\prime}, \beta_{T}^{\prime}\right)$ pairs coincide with each other as seen in Section 2.2. Therefore, there is no interaction between the $\varphi_{\theta}$ mode I and $\varphi_{\beta}$ mode II. Consequently, the mixed total $G$ is simply partitioned as

$$
G_{I}=\alpha_{\theta}^{2} G_{\theta} \text { and } G_{I I}=\alpha_{\beta}^{2} G_{\beta}
$$

The $G_{I}$ or effectively $G_{\theta}$ comes solely from the difference of the two crack tip rotations as seen from Eqs. (16) and (17). This arises from the through thickness shear strains caused by the crack tip opening force $F_{n B \theta}$ which is the shearing force in the thickness direction. Both the crack tip opening force $F_{n B \theta}$ and opening displacement $D_{o p \theta}$ vary with the variation of shear modulus $G_{x z}$. However, the energy release rate $G_{\theta}$ remains constant. $F_{n B \theta}$ can be determined by using Eqs. (17) and (27) with crack closure technique. It is given by Eq. (31).

$$
F_{n B \theta}=h_{1}^{-1}\left(\frac{2 M_{n m \theta}}{\eta}\right)^{1 / 2}
$$


where $\eta=E /\left(6 k^{2} G_{x z}\right)$ and $M_{n m \theta}=4 \gamma^{2} /(1+\gamma)^{2}$ is the resultant moment of the normal interface stress $\sigma_{n}$ from Eq. (6). It is seen that $F_{n B \theta}$ is finite. Therefore, $F_{s B \theta}$ is equal to zero from Eq. (25) as expected since the $\varphi_{\theta}$ mode corresponding to $D_{s h}=0$ coincides with the $\varphi_{\theta_{T}^{\prime}}$ mode corresponding to $F_{s B}=0$. Varying the shear modulus $G_{x z}$ has no effect on $F_{s B \beta}, D_{s h \beta}$ and $D_{o p \beta}$ since there is no interface normal stress in the $\varphi_{\beta}$ mode.

It was mentioned at the end of Section 2.2 that $F D-\left(\theta_{T}^{\prime}, \beta_{T}^{\prime}\right)$ pair approaches to the $D F-(\theta, \beta)$ pair from above and does so asymptotically. Extra care has to be taken in FEM simulation. Very fine meshes are required to ensure that $\beta_{T}^{\prime}$ converges to $\beta$. Otherwise, a $\varphi_{\beta^{\prime}}$ mode can be generated numerically with $\beta=\beta_{T}^{\prime}<\beta^{\prime}<\beta_{E}^{\prime}=\gamma^{3}$.

Partition of a mixed mode based on Euler beam theory is not as straight forward as that in Timoshenko beam theory due to the interactions between the $\varphi_{\theta}$ mode I and $\varphi_{\beta}$ mode II. The interactions are the work done by the crack tip forces $F_{n B \theta}$ and $F_{s B \theta}$ of $\varphi_{\theta}$ mode I on the crack displacements $D_{o p \beta}$ and $D_{s h \beta}$ of $\varphi_{\beta}$ mode II. They are calculated as

$$
W_{I}=\frac{F_{n B \theta} D_{o p \beta}}{2}=\frac{F_{n B \theta}}{4 E}\left(\frac{1}{I_{1}}-\frac{\beta}{I_{2}}\right) d a^{2}
$$

and

$$
W_{I I}=\frac{F_{s B \theta} D_{s h \beta}}{2}=\frac{F_{s B \theta}}{4 E}\left(\frac{h_{1}}{I_{1}}+\frac{\beta h_{2}}{I_{2}}\right) d a
$$

Using Eq. (25), it can be shown that $W_{I}+W_{I I}=0$. That is, the interactions do not produce any net work as expected since the two modes are orthogonal to each other. However, the stealthy interactions cause energy flow between the two modes resulting changes of mode partitions. $F_{n B \theta}$ can be determined by using Eq. (17) and (27) with crack closure technique. It is given in Eq. (34).

$$
F_{n B \theta} d a=2 M_{n m \theta}
$$

From Eq. (25) the crack tip shear force $F_{s B \theta}$ is found as

$$
F_{s B \theta}=\frac{6 \gamma(1-\gamma)}{h_{1}(1+\gamma)^{2}}
$$

It is seen that $F_{s B \theta}$ is of the same value as the resultant interface shear force $F_{s \theta}$ given by Eq. (5). Of course, $F_{s B \theta}$ is no longer zero as in Timoshenko beam theory. $F_{n B \theta}$ is of infinite value here in Euler beam theory instead of a finite value as in Timoshenko beam theory. Substituting Eq. (34) into Eq. (32) gives

$$
\Delta G_{\theta \beta}=\frac{W_{I}}{b d a}=\frac{72 \gamma(\gamma-1)}{E b^{2} h_{1}^{3}(1+\gamma)(1+3 \gamma)}=\frac{3(\gamma-1)}{1+3 \gamma} G_{\theta}
$$

Therefore, a mixed mode is finally partitioned as

$$
G_{I}=\alpha_{\theta}^{2} G_{\theta}+\alpha_{\theta} \alpha_{\beta} \Delta G_{\theta \beta}
$$

and 


$$
G_{I I}=\alpha_{\beta}^{2} G_{\beta}-\alpha_{\theta} \alpha_{\beta} \Delta G_{\theta \beta}
$$

It results in the second pair pure modes $F D-\left(\theta_{E}^{\prime}, \beta_{E}^{\prime}\right)=\left(-1, \gamma^{3}\right)$. It is easy to show that the crack tip shear force $F_{s B \theta_{E}^{\prime}}$ is zero. That is, $F D-\left(\theta_{E}^{\prime}, \beta_{E}^{\prime}\right)=\left(-1, \gamma^{3}\right)$ is also a locally pure pair as said at the end of Section 2.2.

It is worth noting that problems can arise when using FEM simulation based on Euler beam theory due to non-compatibility of displacement at the interface. For example, when using Euler beam element with point interface springs, a numerically 'pure' pair mode $F D-\left(\theta^{\prime}, \beta^{\prime}\right)$ different from $F D-\left(\theta_{E}^{\prime}, \beta_{E}^{\prime}\right)=\left(-1, \gamma^{3}\right)$ will be generated with $\theta<\theta^{\prime}<\theta_{E}^{\prime}=-1$ and $\beta<\beta^{\prime}<\beta_{E}^{\prime}=\gamma^{3}[15-$ 21]. It is also worth noting that $F D-\left(\theta^{\prime}, \beta^{\prime}\right)$ can be generated in experimental tests.

Finally, it is worth noting the following point. When the whole $\Delta a$ region is considered in the evaluation of energy release rate partitions, the through thickness shear effect at the crack tip due to the normal stress $\sigma_{n}$, arising from the $\varphi_{\theta}$ mode I, will disappear. Therefore, for any values of the shear modulus, the energy release rate partitions will remain the same as those for infinitely large shear modulus. That is, the second pair of locally pure modes $F D-\left(\theta_{T}^{\prime}, \beta_{T}^{\prime}\right)$ will disappear and the partitions are the same as that based on Euler beam theory. That is why the $F D$ $\left(\theta_{E}^{\prime}, \beta_{E}^{\prime}\right)=\left(-1, \gamma^{3}\right)$ pair is the second globally pure mode pair in Timoshenko beam theory as mentioned at the end of Section 2.2. It is not surprising that much confusion has been caused by the complex entanglements of the problem in both theoretical and numerical aspects, such as globally pure, locally pure, numerically pure, stealthy interactions, crack tip running contact, etc..

In reference [1], $F D-\left(\theta_{E}^{\prime}, \beta_{E}^{\prime}\right)=\left(-1, \gamma^{3}\right)$ pair is correctly given. The method in [1] is only applicable to symmetric DCBs because it missed the stealthy interactions. In reference [2], crack interface opening stress is ignored. Instead, a crack tip interface bending moment is introduced. Therefore, the mixed mode partition is a kind of global procedure. Moreover, the procedure is a combined numerical and analytical method. It cannot even produce the Williams' modes. The present orthogonal pure mode approach is the key to achieve a correct mixed mode partition.

\subsection{An averaged fracture mode partition rule}

In Section 2.3, mixed mode partition theories have been established based on Euler and Timoshenko beam theories. A detailed study of fracture mode partition spaces is given in the Appendix. An averaged mixed mode partition theory is given in this section based on the combination of Euler beam rules in Eqs. (37) and (38), and Timoshenko beam rules in Eq. (30). Within the context of Timoshenko beam theory there is no interaction between the $\varphi_{\theta}$ mode I and $\varphi_{\beta}$ mode II. On the other hand, there is a full interaction between the $\varphi_{\theta}$ mode I and $\varphi_{\beta}$ mode II in Euler beam theory. Within the first quadrant of the partition space of $\left(\alpha_{\theta}, \alpha_{\beta}\right)$, i.e. between $M_{2 B}=M_{2}=\theta$ and $M_{2 B}=M_{2}=\beta$, as shown in Figs. A1 and A2, the interaction causes energy flow from mode II to mode I as shown in Eqs. (37) and (38). The Euler $G_{I}$ is larger than the Timoshenko $G_{I}$ by the amount of $\alpha_{\theta} \alpha_{\beta} \Delta G_{\theta \beta}$ while the Euler $G_{I I}$ is smaller than the Timoshenko $G_{I I}$ by the same amount. Within the second quadrant of the partition space of $\left(\alpha_{\theta}, \alpha_{\beta}\right)$, i.e. between $M_{2 B}=M_{2}=\beta$ and $M_{2 B}=M_{2}=\gamma^{3}$, and the fourth quadrant, i.e. between $M_{2 B}=M_{2}=-\infty$ and $M_{2 B}=M_{2}=\theta$, the interaction causes energy flow from mode I to mode II. The Euler $G_{I}$ is smaller than the Timoshenko $G_{I}$ by the amount of $\alpha_{\theta} \alpha_{\beta} \Delta G_{\theta \beta}$ while the Euler $G_{I I}$ is larger than the Timoshenko $G_{I I}$ by the same amount. It is reasonable to suggest that the Euler and Timoshenko partition rules act as either upper or lower bounds of partitions. Therefore, 
the average of the two rules is expected to give comparable predictions with plane stress 2D FEM simulations. That is, the amount of energy flow between mode I and mode II is halved, i.e. $\alpha_{\theta} \alpha_{\beta} \Delta G_{\theta \beta} / 2$. Therefore, the averaged theory gives the following partitions

$$
G_{I}=\alpha_{\theta}^{2} G_{\theta}+\alpha_{\theta} \alpha_{\beta} \Delta G_{\theta \beta} / 2
$$

and

$$
G_{I I}=\alpha_{\beta}^{2} G_{\beta}-\alpha_{\theta} \alpha_{\beta} \Delta G_{\theta \beta} / 2
$$

\subsection{Addition of axial forces}

In the above development, the axial forces are not considered for the sake of simplicity and focusing on important mechanical understandings. Now, they are considered in this section. Two axial forces, i.e. $N_{1 B}$ and $N_{2 B}$, are assumed to act on the crack tips of the two beams, respectively. They are positive when in tensile sense. The energy release rate is therefore,

$$
G=\frac{1}{b} \frac{\partial U}{\partial a}=\frac{1}{2 b E}\left(\frac{M_{1 B}^{2}}{I_{1}}+\frac{M_{2 B}^{2}}{I_{2}}-\frac{M_{B}^{2}}{I}+\frac{N_{1 B}^{2}}{A_{1}}+\frac{N_{2 B}^{2}}{A_{2}}-\frac{N_{B}^{2}}{A}\right)
$$

in which

$$
N_{B}=N_{1 B}+N_{2 B}
$$

and

$$
M_{B}=M_{1 B}+M_{2 B}+\left(h_{1} N_{2 B}-h_{2} N_{1 B}\right) / 2
$$

It appears that the energy release rate is now a quadratic form with four variables. In fact only three variables are necessary. Since uniform axial stress does not produce any energy release rate, an effective axial force can be defined as

$$
N_{1 B e}=N_{1 B}-N_{2 B} A_{1} / A_{2}
$$

Then, the energy release rate in Eq. (41) becomes

$$
G=\frac{1}{2 b E}\left[\frac{M_{1 B}^{2}}{I_{1}}+\frac{M_{2 B}^{2}}{I_{2}}-\frac{1}{I}\left(M_{1 B}+M_{2 B}-\frac{h_{2} N_{1 B e}}{2}\right)^{2}+\left(\frac{1}{A_{1}}-\frac{1}{A}\right) N_{1 B e}^{2}\right]
$$

The resultant shear force $F_{s}$ and moment $M_{n}$ in Eqs. (5) and (6) change to be

$$
F_{s}=\frac{6 \gamma}{h_{1}(1+\gamma)^{3}}\left(M_{1 B}+M_{2 B}\right)+\frac{\gamma\left(1+\gamma^{3}\right)}{(1+\gamma)^{4}} N_{1 B e}
$$

and

$$
M_{n}=M_{n m}=\frac{1+3 \gamma}{(1+\gamma)^{3}}\left(\beta M_{1 B}-M_{2 B}\right)+\frac{h_{1} \gamma^{2}(1-\gamma)}{2(1+\gamma)^{3}} N_{1 B e}
$$

respectively.

Since the $D F$ pure pairs are used in the mixed mode partitions, they are considered first. Equating $M_{n}$ to zero gives the following pure mode II condition.

$$
2(1+3 \gamma)\left(\beta M_{1 B}-M_{2 B}\right)+h_{1} \gamma^{2}(1-\gamma) N_{1 B e}=0
$$


The case $M_{1 B}=M_{2 B}=0$ is considered first to examine the role of axial force alone. When $h_{1}=h_{2}$, any $N_{1 B}$ and $N_{2 B}$ produce pure mode II. When $h_{1} \neq h_{2}, N_{1 B e}$ has to be zero, resulting in a uniform stress on the cross section and leading to zero energy release rate. Therefore, it can be concluded that in general $N_{1 B}$ and $N_{2 B}$ alone cannot produce $D F$ pair pure mode II. However, when $M_{1 B}$ and $M_{2 B}$ are present, they have essential effects on the energy release rate through changing the shear stress singularity at the crack tip. Generally, condition Eq. (48) provides two independent pure mode II modes. Here, the following two are chosen for the sake of direct connection with Eq. (9),

$$
\varphi_{\beta_{1}}=\left\{\begin{array}{c}
1 \\
\beta_{1} \\
0
\end{array}\right\}=\left\{\gamma^{2}(3+\gamma) /(1+3 \gamma)\right\} \text { and } \varphi_{\beta_{2}}=\left\{\begin{array}{c}
1 \\
0 \\
\beta_{2}
\end{array}\right\}=\left\{\begin{array}{c}
1 \\
0 \\
2(3+\gamma) /\left[h_{1}(\gamma-1)\right]
\end{array}\right\}
$$

When $h_{1}=h_{2}$

$$
\varphi_{\beta_{2}}=\left\{\begin{array}{c}
0 \\
0 \\
\beta_{2}
\end{array}\right\}=\left\{\begin{array}{l}
0 \\
0 \\
1
\end{array}\right\}
$$

Note that here $\beta_{1}$ is used to replace the previous $\beta$.

The pure mode I condition i.e. Eq. (21) changes to be

$$
D_{s h}=\frac{1}{2 E}\left[\frac{h_{1} M_{1 B}}{I_{1}}+\frac{h_{2} M_{2 B}}{I_{2}}+\frac{2 N_{1 B e}}{A_{1}}\right] d a=0
$$

That is,

$$
6\left(\gamma^{2} M_{1 B}+M_{2 B}\right)+h_{1} \gamma^{2} N_{1 B e}=0
$$

It provides two independent pure mode I modes. Here, the following two are chosen for the sake of direct connection with the Eq. (10),

$$
\varphi_{\theta_{1}}=\left\{\begin{array}{c}
1 \\
\theta_{1} \\
0
\end{array}\right\}=\left\{\begin{array}{c}
1 \\
-\gamma^{2} \\
0
\end{array}\right\} \text { and } \varphi_{\theta_{2}}=\left\{\begin{array}{l}
1 \\
0 \\
\theta_{2}
\end{array}\right\}=\left\{\begin{array}{c}
1 \\
0 \\
-6 / h_{1}
\end{array}\right\}
$$

Here $\theta_{1}$ is used to replace the previous $\theta$. Note that mode $\varphi_{\theta_{2}}$ is independent of $\gamma$. It can be shown that the two conditions (48) and (51) are orthogonal to each other with respect to the coefficient matrix of the energy release rate. Therefore, the two pure mode II modes $\varphi_{\beta_{1}}$ and $\varphi_{\beta_{2}}$ in Eq. (49) are orthogonal to both of the two pure mode I modes $\varphi_{\theta_{1}}$ and $\varphi_{\theta_{2}}$ in Eq. (52). Also, note that $N_{1 B}$ and $N_{2 B}$ alone cannot produce $D F$ pair pure mode I.

For a given mixed mode $M_{1 B}, M_{2 B}$ and $N_{1 B e}$ the mode partition can be done by using any three of the four independent modes. Here, the modes $\varphi_{\theta_{1}}, \varphi_{\beta_{1}}$ and $\varphi_{\beta_{2}}$ are chosen for the partition. Therefore, the partition coefficients can be determined through 


$$
\left\{\begin{array}{l}
M_{1 B} \\
M_{2 B} \\
N_{1 B e}
\end{array}\right\}=\left[\begin{array}{ccc}
1 & 1 & 0 \\
\theta_{1} & \beta_{1} & 0 \\
0 & 0 & 1
\end{array}\right]\left\{\begin{array}{l}
\alpha_{\theta_{1}} \\
\alpha_{\beta_{1}} \\
\alpha_{\beta_{2}}
\end{array}\right\}
$$

for $h_{1}=h_{2}$ and

$$
\left\{\begin{array}{l}
M_{1 B} \\
M_{2 B} \\
N_{1 B e}
\end{array}\right\}=\left[\begin{array}{ccc}
1 & 1 & 1 \\
\theta_{1} & \beta_{1} & 0 \\
0 & 0 & \beta_{2}
\end{array}\right]\left\{\begin{array}{l}
\alpha_{\theta_{1}} \\
\alpha_{\beta_{1}} \\
\alpha_{\beta_{2}}
\end{array}\right\}
$$

for $h_{1} \neq h_{2}$. From Eq. (54), the partition coefficients for the case of $h_{1} \neq h_{2}$ are given by

$$
\alpha_{\beta_{2}}=\frac{N_{1 B e}}{\beta_{2}} \text { and }\left\{\begin{array}{l}
\alpha_{\theta_{1}} \\
\alpha_{\beta_{1}}
\end{array}\right\}=\frac{1}{\beta_{1}-\theta_{1}}\left\{\begin{array}{c}
\beta_{1}\left(M_{1 B}-\alpha_{\beta_{2}}\right)-M_{2 B} \\
-\theta_{1}\left(M_{1 B}-\alpha_{\beta_{2}}\right)+M_{2 B}
\end{array}\right\}
$$

Then, the mode I energy release rate $G_{I}$ is calculated as

$$
G_{I}=\alpha_{\theta_{1}}^{2} G_{\theta_{1}}+\alpha_{\theta_{1}} \alpha_{\beta_{1}} \Delta G_{\theta_{1} \beta_{1}}+\alpha_{\theta_{1}} \alpha_{\beta_{2}} \Delta G_{\theta_{1} \beta_{2}}
$$

Within the context of Timoshenko beam theory, the second and third terms simply disappear. Within the context Euler beam theory, the term $\Delta G_{\theta_{1} \beta_{1}}$ by given in Eq. (36) and the term $\Delta G_{\theta_{1} \beta_{2}}$ is calculated as

$$
\Delta G_{\theta_{1} \beta_{2}}=\frac{24 \gamma^{2}}{E b^{2} h_{1}^{3}(1+\gamma)^{2}}=\frac{\gamma}{1+\gamma} G_{\theta_{1}}
$$

for $h_{1} \neq h_{2}$. For $h_{1}=h_{2}$ then $\Delta G_{\theta_{1} \beta_{2}}=0$. The mode II energy release rate is simply $G_{I I}=G-G_{I}$. In the averaged rule, these interaction terms are halved.

Next, it may be interesting to look at the $F D$ pure pairs. Since the $F D$ pure pairs coincide with the $D F$ pure pairs in Timoshenko beam theory, we only need to look at the $F D$ pure pairs in Euler beam theory, which are globally pure pairs in Timoshenko beam theory. This can be done by looking at the zero resultant shear force condition. That is, from Eq. (46), $F_{s}=0$ gives

$$
6(1+\gamma)\left(M_{1 B}+M_{2 B}\right)+h_{1}\left(1+\gamma^{3}\right) N_{1 B e}=0
$$

which yields two independent $F D$ pair pure modes I which are

$$
\varphi_{\theta_{1 E}^{\prime}}=\left\{\begin{array}{c}
1 \\
\theta_{1 E}^{\prime} \\
0
\end{array}\right\}=\left\{\begin{array}{c}
1 \\
-1 \\
0
\end{array}\right\} \text { and } \varphi_{\theta_{2 E}^{\prime}}=\left\{\begin{array}{c}
1 \\
0 \\
\theta_{2 E}^{\prime}
\end{array}\right\}=\left\{\begin{array}{c}
1 \\
0 \\
-6(1+\gamma) /\left[h_{1}\left(1+\gamma^{3}\right)\right]
\end{array}\right\}
$$

Here $\theta_{1 E}^{\prime}$ is used to replace the previous $\theta_{E}^{\prime}$. Similar to $\varphi_{\theta_{1 E}^{\prime}}$, the mode $\varphi_{\theta_{2 E}^{\prime}}$ is also a globally and locally pure mode $\mathrm{I}$, that is, both the resultant shear force $F_{s \theta_{2 E}^{\prime}}$ in the $\Delta a$ region and crack tip shear force $F_{s B \theta_{2 E}^{\prime}}$ are zero within the context of Euler beam theory. It is only globally pure within the context of Timoshenko beam theory, that is, only the resultant shear force $F_{s \theta_{2 E}^{\prime}}$ is zero. However, although $\varphi_{\theta_{1 E}^{\prime}}$ still has its orthogonal partner $\varphi_{\beta_{1 E}^{\prime}}$ corresponding to $D_{o p \beta_{1 E}^{\prime}}=0$, $\varphi_{\theta_{2 E}^{\prime}}$ has 
no orthogonal pair partner $\varphi_{\beta_{2 E}^{\prime}}$ corresponding to $D_{o p \beta_{2 E}^{\prime}}=0$ since crack tip opening displacement does not contain axial forces, as shown in Eq. (11). Again, note that $N_{1 B}$ and $N_{2 B}$ alone cannot produce $F D$ pair pure mode I. However, $N_{1 B}$ and $N_{2 B}$ alone can produce $F D$ pair pure mode II in Euler beam theory corresponding to $D_{o p}=0$.

\subsection{Addition of Shear Forces}

Two shear forces, i.e. $P_{1 B}$ and $P_{2 B}$, are assumed to act on the crack tips of the two beams, respectively. They are positive when upwards. The resultant shear force $F_{s}$ and moment $M_{n}$ in expressions in (5) and (6) change to be

$$
F_{s}=\frac{6 \gamma}{h_{1}(1+\gamma)^{3}}\left[M_{1 B}+M_{2 B}+\left(P_{1 B}+P_{2 B}\right) \Delta a\right]+\frac{\gamma\left(1+\gamma^{3}\right)}{(1+\gamma)^{4}} N_{1 B e}
$$

and

$$
M_{n}=M_{n m}+F_{n} \Delta a=\frac{1+3 \gamma}{(1+\gamma)^{3}}\left(\beta_{1} M_{1 B}-M_{2 B}\right)+\frac{h_{1} \gamma^{2}(1-\gamma)}{2(1+\gamma)^{3}} N_{1 B e}+F_{n} \Delta a
$$

respectively. In Eq. (61) the resultant normal interface force $F_{n}$ is given by

$$
F_{n}=b \int_{0}^{\Delta a} \sigma_{n} d x=\frac{1+3 \gamma}{(1+\gamma)^{3}}\left(\beta_{1} P_{1 B}-P_{2 B}\right)
$$

in Euler beam theory and

$$
F_{n}=b \int_{0}^{\Delta a} \sigma_{n} d x=\frac{1}{1+\gamma}\left(\gamma P_{1 B}-P_{2 B}\right)
$$

in Timoshenko beam theory. The crack tip shearing displacement becomes

$$
D_{s h}=\frac{1}{2 E}\left[\left(\frac{h_{1} M_{1 B}}{I_{1}}+\frac{h_{2} M_{2 B}}{I_{2}}\right) d a-\left(\frac{h_{1} P_{1 B}}{I_{1}}+\frac{h_{2} P_{2 B}}{I_{2}}\right) \frac{d a^{2}}{2}+\left(\frac{2 N_{1 B e}}{A_{1}}\right) d a\right]
$$

By neglecting the higher order $P_{1 B}$ and $P_{2 B}$ term, $D_{s h}=0$ remains the same as Eq. (51). Therefore, we have the same $\varphi_{\theta_{1}}$ and $\varphi_{\theta_{2}}$ as those in Eq. (52). Since $\Delta a$ is arbitrary, $M_{n}=0$ requires both $M_{n m}=0$ and $F_{n}=0$. Therefore, Eq. (48) is still valid and we have the same $\varphi_{\beta_{1}}$ and $\varphi_{\beta_{2}}$ as that in Eqs. (49) and (50). $F_{n}=0$ requires that $P_{1 B}$ and $P_{2 B}$ satisfy $P_{2 B}=\beta_{1} P_{1 B}$ in Euler beam theory and $P_{2 B}=\gamma P_{1 B}$ in Timoshenko beam theory. These two relationships are the normal shear forces distributions in the respective beam theories. Since $P_{1 B}$ and $P_{2 B}$ have no contribution to strain energy in Euler beam theory we only consider their contributions to mixed mode partition in Timoshenko beam theory.

Since through-thickness shear effect does not generate any axial displacement in Timoshenko beam theory, the two crack tip forces $P_{1 B}$ and $P_{2 B}$ produce pure mode I fracture only. Here, it is designated as $P$ mode $\mathrm{I}$. The crack tip opening displacement $D_{o p P}$ is given by

$$
D_{o p P}=\frac{\left(\gamma P_{1 B}-P_{2 B}\right) d a}{\gamma b h_{1} k^{2} G_{x z}}
$$


Within the $\Delta a$ region as shown in Fig. 2, the through-thickness shearing Eqs. (12) and (13) change to be

$$
k^{2} G_{x z} A_{1}\left(w_{1}^{(1)}-\psi_{1}\right)=b \int_{0}^{x} \sigma_{n} d x-P_{1 B}
$$

and

$$
k^{2} G_{x z} A_{2}\left(w_{2}^{(1)}-\psi_{2}\right)=-b \int_{0}^{x} \sigma_{n} d x-P_{2 B}
$$

for beams 1 and 2, respectively. Using the same way to get Eq. (16), Eq. (68) is obtained.

$$
\psi_{1 B}-\psi_{2 B}=\frac{1}{k^{2} G_{x z}}\left[\left(\frac{P_{1 B}}{A_{1}}-\frac{P_{2 B}}{A_{2}}\right)-F_{n B}\left(\frac{1}{A_{1}}+\frac{1}{A_{2}}\right)\right]
$$

In the presence of $P_{1 B}$ and $P_{2 B}$ alone, $\psi_{1 B}-\psi_{2 B}=0$. Therefore, crack tip opening force $F_{n B P}$ in the $P$ mode is

$$
F_{n B P}=\frac{\gamma P_{1 B}-P_{2 B}}{1+\gamma}
$$

which has the same value as the resultant normal force $F_{n}$ in Eq. (63). Therefore, the energy release rate of $P$ mode I, i.e. $G_{P}$ is obtained in Eq. (70) from Eqs. (65) and (69) by using crack closure technique.

$$
G_{P}=\frac{\left(\gamma P_{1 B}-P_{2 B}\right)^{2}}{2 b^{2} h_{1} k^{2} G_{x z} \gamma(1+\gamma)}
$$

It is easy to show that $G_{P}$ above is equal to

$$
G_{P}=\frac{1}{2 b k^{2} G_{x z}}\left[\frac{P_{1 B}^{2}}{A_{1}}+\frac{P_{2 B}^{2}}{A_{2}}-\frac{\left(P_{1 B}+P_{2 B}\right)^{2}}{A}\right]
$$

Using Eqs. (31) and (65), the interaction between the $\varphi_{\theta_{1}}$ mode I and the $P$ mode I is determined as

$$
\Delta G_{\theta_{1} P}=\frac{4 \sqrt{3}\left(\gamma P_{1 B}-P_{2 B}\right)}{b^{2} h_{1}^{2}(1+\gamma)\left(k^{2} G_{x z} E\right)^{1 / 2}}
$$

It can be shown that the same relationship as that in Eq. (25) can be obtained between the crack tip opening force $F_{n B P}$ and crack tip shearing $F_{s B P}$ in $P$ mode I. Since $F_{n B P}$ given in Eq. (69) is of a finite value, then $F_{s B P}$ is zero. Moreover, $D F$ and $F D$ pure pairs coincide with each other in Timoshenko beam theory. Therefore, the $P$ mode I has no interaction with $\varphi_{\beta_{1}}$ and $\varphi_{\beta_{2}}$ modes. Consequently, within the context of Timoshenko beam theory, the mode I energy release rate is given by

$$
G_{I}=\alpha_{\theta_{1}}^{2} G_{\theta_{1}}+G_{P}+\alpha_{\theta_{1}} \Delta G_{\theta_{1} P}
$$

The mode II energy release rate is the same as before. In the averaged rule, half of the interaction terms, i.e. $\left(\alpha_{\theta_{1}} \alpha_{\beta_{1}} \Delta G_{\theta_{1} \beta_{1}}+\alpha_{\theta_{1}} \alpha_{\beta_{2}} \Delta G_{\theta_{1} \beta_{2}}\right) / 2$ is added to $G_{I}$ in Eq. (73) and is deducted from $G_{I I}$. 
Finally, it is seen from Eq. (60) that when $P_{1 B}+P_{2 B}=0$, the same $\varphi_{\theta_{1 E}^{\prime}}$ and $\varphi_{\theta_{2 E}^{\prime}}$ modes as those in Eq. (59) are obtained. However, when $P_{1 B}+P_{2 B} \neq 0$, there is no Euler FD pure pair.

\subsection{Summary of mixed mode partition formulae}

The above thorough and comprehensive development reveals the in depth mechanics of mixed mode partitions. It is extremely important for thorough understanding of the problem. Within the context of Euler beam theory, there are two sets of distinct pure mode pairs. They are $D F-(\theta, \beta)$ set pairs and $F D-\left(\theta_{E}^{\prime}, \beta_{E}^{\prime}\right)$ set pairs. Within the context of Timoshenko beam theory, the two sets coincide at $D F-(\theta, \beta)$ set pairs. The $D F-(\theta, \beta)$ set pairs form a complete basis for mix mode partitions. Stealthy interactions need to be considered in Euler beam theory while there are no stealthy interactions in Timoshenko beam theory. While all the above partition formulae serve the best to understand the mechanics of partition, a more convenient form of them can be obtained in what follows. Since there are $D F-(\theta, \beta)$ set pairs and $F D-\left(\theta_{E}^{\prime}, \beta_{E}^{\prime}\right)$ set pairs in Euler beam theory, the mix mode partition can be written as

$$
\begin{aligned}
G_{I E} & =C_{I E}\left(M_{1 B}-M_{2 B} / \beta_{1}-N_{1 B e} / \beta_{2}\right)\left(M_{1 B}-M_{2 B} / \beta_{1 E}^{\prime}\right) \\
G_{I I E} & =C_{I I E}\left(M_{1 B}-M_{2 B} / \theta_{1}-N_{1 B e} / \theta_{2}\right)\left(M_{1 B}-M_{2 B} / \theta_{1 E}^{\prime}-N_{1 B e} / \theta_{2 E}^{\prime}\right)
\end{aligned}
$$

Where $C_{I E}$ and $C_{I I E}$ are constants and can be determined by using $\theta_{1}$ and $\beta_{1}$ modes, respectively. They are

$$
\begin{aligned}
& C_{I E}=G_{\theta_{1}}\left(1-\theta_{1} / \beta_{1}\right)\left(1-\theta_{1} / \beta_{1 E}^{\prime}\right) \\
& C_{I I E}=G_{\beta_{1}}\left(1-\beta_{1} / \theta_{1}\right)\left(1-\beta_{1} / \theta_{1 E}^{\prime}\right)
\end{aligned}
$$

In Timoshenko beam theory,

$$
\begin{gathered}
G_{I T}=C_{I T}\left(M_{1 B}-M_{2 B} / \beta_{1}-N_{1 B e} / \beta_{2}\right)^{2}+G_{P}+\alpha_{\theta_{1}} \Delta G_{\theta_{1} P}(78) \\
G_{I I T}=C_{I I T}\left(M_{1 B}-M_{2 B} / \theta_{1}-N_{1 B e} / \theta_{2}\right)^{2}
\end{gathered}
$$

with

$$
\begin{aligned}
& C_{I T}=G_{\theta_{1}}\left(1-\theta_{1} / \beta_{1}\right)^{2} \\
& C_{I I T}=G_{\beta_{1}}\left(1-\beta_{1} / \theta_{1}\right)^{2}
\end{aligned}
$$

In Averaged theory,

$$
\begin{aligned}
& G_{I A}=\left(G_{I E}+G_{I T}\right) / 2+2\left(G_{P}+\alpha_{\theta_{1}} \Delta G_{\theta_{1} P}\right) \\
& G_{I I A}=\left(G_{I I E}+G_{I I T}\right) / 2
\end{aligned}
$$

Obviously, these formulae are equivalent to those in previous sections and more convenient for use. However, they lack physical meanings.

\subsection{Application to more general one-dimensional fracture}

Here, two more general one-dimensional fractures are considered as shown in Figs. 3 and 4. A clamped beam with a central delamination is shown in Fig. 3 and a clamped circular plate with a 
central delamination is shown in Fig. 4. Since space is limited, only a few results are given here with $G_{x z}=\infty$. Details of this part will be reported in the work of authors [20,21]. By using the theory in the previous sections, the pure mode conditions are found to be

$$
P_{2}=\theta_{1} P_{1}
$$

and

$$
P_{2}=\beta_{1} P_{1}
$$

for pure modes I and II, respectively. The analytical energy release rates for the beam in mode I and mode II are given by Eqs. (86) and (87) respectively,

$$
\begin{gathered}
G_{\theta_{1}}=\frac{3 P_{1}^{2} a^{2} \gamma}{E b^{2} h_{1}^{3}(1+\gamma)} \\
G_{\beta_{1}}=\frac{9 P_{1}^{2} a^{2} \gamma(1+\gamma)}{E b^{2} h_{1}^{3}(1+3 \gamma)^{2}}
\end{gathered}
$$

The analytical energy release rates for the plate in mode I and mode II are given by Eqs. (88) and (89) respectively.

$$
\begin{gathered}
G_{\theta_{1}}=\frac{3 P_{1}^{2} \gamma\left(1-v^{2}\right)}{2 E h_{1}^{3} \pi^{2}(1+\gamma)} \\
G_{\beta_{1}}=\frac{9 \gamma P_{1}^{2}\left(1-v^{2}\right)(1+\gamma)}{2 E h_{1}^{3} \pi^{2}(1+3 \gamma)^{2}}
\end{gathered}
$$

\section{Tests}

To validate the theory, an FEM simulation capability has been developed based on the Euler, Timoshenko beam theories and 2D elasticity in conjunction with imaginary normal and shear point springs and the crack closure technique $[22,23]$ to numerically determine the energy release rate and its partitions. A contact algorithm is also implemented to deal with any possible contact in loading. In the following tests, the stiffness of interface springs are taken to be $k_{s}=10^{6}$. However, $k_{s}$ is taken to be $k_{s}=10^{14}$ in the tests in Section 3.5.

\subsection{Tests with crack tip bending moments $M_{1 B}$ and $M_{2 B}$}

The first test aims to examine the present partition rules based Euler beam theory or Timoshenko beam theory with an infinitely large shear modulus. A DCB as shown in Fig. 1 is considered. The Young's modulus is $E=1$ and the Poisson's ratio is $v=0.3$. The intact length is 100 and the width is $b=1$. The thickness is $h=h_{1}+h_{2}=3$ with $h_{1}=1$. Therefore, the thickness ratio is $\gamma=2$. The DCB is under tip bending moments $M_{1}=1$ and $M_{2}$ which varies from -10 to 10. Both analytical and numerical results are presented in Fig. 5 and Table 1.

One set of numerical results in Fig. 5 and Table 1 is from the linear Timoshenko beam element with point interface springs. A large shear modulus $G_{x z}=10^{4}$ is used to simulate the Euler beam theory. To avoid shear locking, the reduced integration technique is applied. It is seen that this set of numerical predictions are identical to the analytical results. Both pairs of pure modes, i.e. $D F-\left(\theta_{1}, \beta_{1}\right)=(-4,20 / 7)$ and $F D-\left(\theta_{1 E}^{\prime}, \beta_{1 E}^{\prime}\right)=(-1,8)$ are predicted. When increasing 
$M_{2}$ further from $M_{2}=\beta_{1 E}^{\prime}=8$, DCB tip contact starts. In the present theory, the fracture mode will be deflected into a mixed mode region between the $\beta_{1}=20 / 7=2.857$ line and $\beta_{1 E}^{\prime}=\gamma^{3}=8$ line immediately as shown in Fig. A2. As given in Eq. (A4b), $k=5>\beta_{1}$, the fracture mode will stay in the mixed mode region with increasing $M_{2}$. This set of numerical predictions agrees very well the present theory.

Another set of numerical results in Fig. 5 and Table 1 is from the cubic Euler beam element with point interface springs. It is seen that the $D F-\left(\theta_{1}, \beta_{1}\right)=(-4,20 / 7)$ pair is accurately predicted while the $F D$ - $\left(\theta_{1 E}^{\prime}, \beta_{1 E}^{\prime}\right)$ pair is predicted as $F D-\left(\theta_{1}^{\prime}, \beta_{1}^{\prime}\right)=(-1.8087,5.2512)$ instead of $F D-\left(\theta_{1 E}^{\prime}, \beta_{1 E}^{\prime}\right)=\left(-1, \gamma^{3}\right)=(-1,8)$. When $M_{2}$ is in the range from $\beta_{1}^{\prime}=5.2512$ to $\gamma^{3}=8$, crack tip running contact occurs resulting in a pure mode II region. When $M_{2}$ increases further from $M_{2}=\gamma^{3}=8$, both crack tip running contact and DCB tip contact occur and the fracture mode is deflected back into the pure mode II region at $M_{2}=\gamma^{3}=8$. Since $k=5<\beta_{1}^{\prime}=5.2512$, the fracture mode will eventually enter the mixed mode region between $\beta_{1}=20 / 7=2.857$ and $\beta_{1}^{\prime}=5.2512$. It is seen that this set of results do not agree with the analytical predictions. As mentioned earlier, the difference is due to the incompatibility of the Euler beam elements at the interface.

In both set of numerical results, 11 identical elements are used in the whole length of each beam with 10 in the intact length and one element in the cracked length. That is, the cracked length is 10 . However, since both types of contact can occur beyond $M_{2}=\gamma^{3}=8$, here the cracked length is 30 and three elements are used to differentiate the crack tip running contact and DCB tip contact in the loading range beyond $M_{2}=8$.

The second test aims to examine the present partition rules based on Timoshenko beam theory with shear modulus of a finite value. The identical DCB as in the first test is considered but with a shear modulus of $G_{x z}=E /[2(1+v)]=1 / 2.6$. The results are presented in Fig. 6 and Table 2 . Two sets of numerical results are obtained from the linear Timoshenko beam elements with reduced integration technique corresponding to a fine mesh $801 \times 2$ and a coarse mesh $201 \times 2$. The fine mesh uses 801 identical elements in the whole length of each beam with 800 in the intact length and one element in the cracked length while the coarse mesh uses 200 elements in the intact length and one element in the cracked length. It is seen that the FEM results converge very well to the present analytical rules. The two pairs of pure modes, i.e. $D F-\left(\theta_{1}, \beta_{1}\right)$ and $F D$ $\left(\theta_{1 T}^{\prime}, \beta_{1 T}^{\prime}\right)$ coincide at $D F-\left(\theta_{1}, \beta_{1}\right)$. Crack tip running contact starts at $M_{2}=\beta_{1}=20 / 7=2.857$ and the fracture mode enters a pure mode II region between $\beta_{1}=20 / 7=2.857$ and $\beta_{1 E}^{\prime}=\gamma^{3}=8$ as shown in Fig. A2. DCB tip contact starts beyond $M_{2}=\beta_{1 E}^{\prime}=8$ and the fracture mode is deflected back into the pure mode II region. As given in Eq. (A4b), $k=5>\beta_{1}$, the fracture mode will stay in the pure mode II region with increasing $M_{2}$. The present theory agrees very well with the FEM predictions. It is worth noting that the region between $\beta_{1}=20 / 7=2.857$ and $\beta_{1 E}^{\prime}=\gamma^{3}=8$ as shown in Fig. A2 is a mixed mode region in Euler beam theory.

It should be noted that a very fine mesh is required to ensure that $F_{n B \theta_{1}} d a$ is negligible in order to get rid of the interactions between $\theta_{1}$ and $\beta_{1}$ modes and avoid a numerically generated second pure pair of modes $F D$ - $\left(\theta_{1}^{\prime}, \beta_{1}^{\prime}\right)$ which is different from $D F-\left(\theta_{1}, \beta_{1}\right)$ with $\theta_{1}<\theta_{1}^{\prime}<-1$ and $\beta_{1}<\beta_{1}^{\prime}<\gamma^{3}$. This can be observed in the predictions by using the coarse mesh $201 \mathrm{x} 2$.

The third test aims to show the local and global pureness of the two pairs, i.e. $D F-\left(\theta_{1}, \beta_{1}\right)$ and $F D-\left(\theta_{1 E}^{\prime}, \beta_{1 E}^{\prime}\right)=\left(-1, \gamma^{3}\right)$ by using FEM based on Euler beam theory, Timoshenko beam theory 
and plane stress elasticity. Multiple interface springs are used in the calculation of energy release rate partitions. The DCB considered is the same as that in test 1 and test 2 . The thickness ratio is $\gamma=2$.

Table 3 records the predictions using cubic Euler beam elements. It is seen that the local and global pureness of the $D F-\left(\theta_{1}, \beta_{1}\right)=(-4,20 / 7)$ pair is well demonstrated. Although the local pureness of the $F D-\left(\theta_{1 E}^{\prime}, \beta_{1 E}^{\prime}\right)=\left(-1, \gamma^{3}\right)=(-1,8)$ pair cannot be predicted due to the incompatibility of displacement at the interface, the global pureness is predicted when using more springs.

Table 4 records the predictions using linear Timoshenko beam elements with a large shear modulus to represent the Euler beam theory. It is seen that both local and global pureness of the two pairs are demonstrated within the context of Euler beam theory.

Table 5 records the predictions using linear Timoshenko beam elements with a normal shear modulus $G_{x z}=1 / 2.6$. It is observed that the local and global pureness of the $D F$ $\left(\theta_{1}, \beta_{1}\right)=(-4,20 / 7)$ pair is always there, however, the $F D-\left(\theta_{1 E}^{\prime}, \beta_{1 E}^{\prime}\right)=\left(-1, \gamma^{3}\right)=(-1,8)$ pair is no longer locally pure although its global pureness is demonstrated.

Table 6 records the predictions using plane stress elements with a normal shear modulus $G_{x z}=1 / 2.6$. In the simulation, 300 identical elements are used in each beam in the intact length and 20 elements in the cracked length. 2 elements are used in the thickness of the upper beam and 4 elements in the lower beam. The local and global pureness of the $D F-\left(\theta_{1}, \beta_{1}\right)=(-4,20 / 7)$ pair is again demonstrated. Again, the $F D-\left(\theta_{1 E}^{\prime}, \beta_{1 E}^{\prime}\right)=\left(-1, \gamma^{3}\right)=(-1,8)$ pair is not locally pure. But, its global pureness is demonstrated.

The fourth test compares the predictions from various approaches including the present Euler beam rule, Timoshenko beam rule, the averaged rule, Hutchinson and Suo [3] rule, and the plane stress FEM. The same DCB is considered with a normal shear modulus $G_{x z}=1 / 2.6$. Results are presented in Fig. 7 and Table 7. In the simulation, a very fine mesh with $320 \times 6$ four-node planestress quadrilateral (QUAD4) elements is used to guarantee accurate converged predictions. 300 identical QUAD4 elements are used in each beam in the intact length and 20 QUAD4 elements in the cracked length. 2 elements are used in the thickness of the upper beam and 4 elements in the lower beam. Fig. 7 and Table 7 show clearly either upper or lower bounds property of present Euler and Timoshenko beam rules. Within the first quadrant of the partition space of $\left(\alpha_{\theta_{1}}, \alpha_{\beta_{1}}\right)$ as shown in Fig. A2, i.e. between $M_{2 B}=\theta_{1}=-4$ and $M_{2 B}=\beta_{1}=20 / 7$, the interaction causes energy flow from mode II to mode I as shown in Eqs. (37) and (38). Therefore, it is seen that the Euler $G_{I}$ is larger than the Timoshenko $G_{I}$ by the amount of $\alpha_{\theta_{1}} \alpha_{\beta_{1}} \Delta G_{\theta_{1} \beta_{1}}$ while the Euler $G_{I I}$ is smaller than the Timoshenko $G_{I I}$ by the same amount. Within the second quadrant of the partition space of $\left(\alpha_{\theta_{1}}, \alpha_{\beta_{1}}\right)$, i.e. between $M_{2 B}=\beta_{1}=20 / 7$ and $M_{2 B}=\gamma^{3}=8$, and the fourth quadrant, i.e. between $M_{2 B}=-\infty$ and $M_{2 B}=\theta_{1}=-4$, it is seen that the Euler $G_{I}$ is smaller than the Timoshenko $G_{I}$ by the amount of $\alpha_{\theta_{1}} \alpha_{\beta_{1}} \Delta G_{\theta_{1} \beta_{1}}$ while the Euler $G_{I I}$ is larger than the Timoshenko $G_{I I}$ by the same amount. The present averaged rule has an excellent agreement with the plane stress FEM predictions. The present Euler beam rule and Timoshenko beam rule are completely analytical rules. They are exact and agree completely with beam FEM predictions. The rule of Hutchinson and Suo [3] is a combined numerical and analytical rule and does not agree with the beam FEM predictions whilst it agrees well with the present averaged rule and the plane stress FEM predictions.

Another point to note is the crack tip running contact. The present Euler rule does not predict it whilst the present Timoshenko rule predicts it occurring at $\beta_{1 T}^{\prime}=\beta_{1}=20 / 7=2.857$ which 
agrees very well with the Timoshenko FEM prediction. Due to the crack tip running contact, a pure mode II region exists between $M_{2 B}=\beta_{1}=20 / 7=2.857$ and $M_{2 B}=M_{2}=\gamma^{3}=8$. The present averaged rule predicts it occurring at $44 / 13=3.385$. The plane stress FEM predicts it at 3.67. The rule of Hutchinson and Suo [3] does not provide this information.

The fifth test is the same as the fourth test except that the thickness ratio is $\gamma=4$. Results are presented in Fig. 8 and Table 8. Again, the either upper or lower bounds property of present Euler and Timoshenko beam rules is shown. The present averaged rule is in good agreement with the plane stress FEM predictions. Also, the rule of Hutchinson and Suo [3] has an excellent agreement with the plane stress FEM predictions.

Again, the present Euler beam rule does not predict crack tip running contact. The present Timoshenko rule predicts it at $M_{2 B}=\beta_{1}=112 / 13=8.615$. The present averaged rule predicts it at $1104 / 51=21.647$. The plane stress FEM predicts it at 13.82 . It is seen that predictions differ a lot from different theories for large $\gamma$. However, it is observed that the 2D FEM predictions for $G_{I}$ are very close to zero between $M_{2}=8.615$ and $M_{2}=13.82$. Therefore, it is seen that the present Timoshenko beam rule predicts the pure mode II region very well in comparison with the 2D FEM predictions.

\subsection{Tests with crack tip bending moment $M_{1 B}$ and crack tip axial force $N_{1 B}$}

The DCB considered here is the same as that in Section 3.1 with shear modulus $G_{x z}=1 / 2.6$ and $\gamma=2$. The DCB is under a tip bending moment $M_{1}=1$ and an axial force $N_{1}$ which varies from -10 to 10 . The predictions from the present Euler, Timoshenko, averaged rules and plane stress FEM are shown in Fig. 9 and Table 9. It is seen that the averaged rule has an excellent agreement with the plane stress FEM results. The Euler and Timoshenko rules have almost identical predictions with the corresponding Euler and Timoshenko FEM results which are not shown in the Fig. 9 and Table 9. The pure $D F-\left(\theta_{2}, \beta_{2}\right)=(-6,10)$ pair modes are exactly predicted by Eqs. (52) and (49). The $\theta_{2 E}^{\prime}=-2$ is also exactly predicted as Eq. (59). Its orthogonal partner $\beta_{2 E}^{\prime}$ does not exist as the theory predicts.

\subsection{Tests with crack tip axial forces $N_{1 B}$ and $N_{2 B}$}

The DCB considered here is the same as that in Section 3.1 with shear modulus $G_{x z}=1 / 2.6$ and $\gamma=2$. The DCB is under tip axial forces $N_{1}=1$ and $N_{2}$ varying from -10 to 10 . The predictions from the present Euler, Timoshenko, averaged rules and plane stress FEM are shown in Fig. 10 and Table 10. The Euler beam rule gives pure mode II while the Timoshenko beam rule gives mixed modes with a constant mixing ratio as expected since there is effectively only one load parameter, i.e. $N_{1 B e}$. It is easy to show that $G_{I} / G=3\left[(1+\gamma)\left(1-\gamma^{2}\right)\right] /\left[4\left(1+\gamma^{3}\right)\right]$. The averaged rule agrees well with plane stress FEM predictions. Note that energy release rate $G$ is zero when $N_{2}=\gamma N_{1}=2$.

\subsection{Tests with crack tip bending moments $M_{1 B}, M_{2 B}$ and crack tip shear forces $P_{1 B}, P_{2 B}$}

The first test in this section aims to demonstrate the $P$ mode I and its interaction with $\theta_{1}$ mode I. The DCB in Section 3.1 is considered again. Here, the cracked length $a=10$ is specified. Two values of through thickness shear modulus are used, i.e. $G_{x z}=1 / 2.6$ and $1 / 26$. Two DCB tip 
shear forces are applied, that is, $P_{1}=1$ and $P_{2}=\theta P_{1}=-4$. The analytical and numerical results are recorded in Table 11. A good agreement is observed.

The next test in this section is to show the comparisons between the present theories and FEM simulations. The DCB tip force $P_{1}$ is fixed at 1 and $P_{2}$ varies from -10 to 10 . The results are presented in Fig. 11 and Table 12. It is observed that the Timoshenko beam FEM predictions agree very well with the present partition rules based on Timoshenko beam theory while the 2D FEM predictions agree very well with present averaged partition rule. The rule of Hutchinson and Suo [3] is not able to consider this case.

\subsection{Tests with fully clamped beams and axisymmetric plates}

The tests in this section are concerned with pure modes in the more general one-dimensional fracture shown in Figs. 3 and 4. In this section, the stiffness of interface springs are taken to be $k_{s}=10^{14}$. Table 13 shows the data of the beam in Fig. 3 .

Only the $D F-(\theta, \beta)$ pure pairs are considered due to space limitations. Details of this part will be reported in the work of authors $[20,21]$. The loads are set to be $P_{1}=1 \mathrm{~N}$ and $P_{2}=-4 \mathrm{~N}$ for $\varphi_{\theta_{1}}$ mode I and $P_{2}=20 / 7 \mathrm{~N}$ for $\varphi_{\beta_{1}}$ mode II. The results from Eqs. (86) and (87) and Timoshenko beam FEM with $G_{x z}=10^{16} \mathrm{~N} / \mathrm{m}^{2}$ are given in Table 14. These values are for the total clamped beam, i.e. both cracks combined. An excellent agreement is observed between the analytical and numerical results.

Table 15 gives the data of the axisymmetric plate shown in Fig. 4. Again, the loads are set to be $P_{1}=1 \mathrm{~N}$ and $P_{2}=-4 \mathrm{~N}$ for $\varphi_{\theta_{1}}$ mode I and $P_{2}=20 / 7 \mathrm{~N}$ for $\varphi_{\beta_{1}}$ mode II. Table 16 records the results from Eqs. (88) and (89) and axisymmetric ring FEM based on first order shear deformable plate theory with $G_{x z}=10^{16} \mathrm{~N} / \mathrm{m}^{2}$. Excellent agreement is again observed between the analytical and numerical results.

\section{Conclusions}

Due to its in-depth subtleness and complex entanglements, the DCB fracture problem has caused considerable confusion. Analytical theories are developed in this paper based on Euler and Timoshenko beam theories using a brand new approach which reveals the hidden mechanics of the problem. Both Euler and Timoshenko beam theories have the same $D F$ pairs of pure modes. The $F D$ pairs in Timoshenko beam theory coincide with the $D F$ pairs, but are only locally pure. The $F D$ pairs in Euler beam theory, when they exist, are different from the $D F$ pairs. $D F$ pairs are both locally and globally pure in both Euler and Timoshenko beam theories whilst the $F D$ pairs in Euler beam theory, when they exist, are both locally and globally pure in Euler beam theory and only globally pure in Timoshenko beam theory. The $D F$ pairs form a complete basis for mixed mode partitions. Stealthy interactions exist between the $D$ pure mode I modes and $F$ pure mode II modes in Euler beam theory, which disappear in Timoshenko beam theory. Axial forces alone produce a pure mode II in Euler beam theory and mixed mode in Timoshenko beam theory. Through-thickness shear forces at the crack tip produce pure mode I. The Euler and Timoshenko beam partition theories agree very well with the corresponding beam FEM predictions. An averaged partition theory is established by averaging the Euler and Timoshenko beam partitions. It agrees well with 2D FEM predictions. The fracture mode partition space is thoroughly investigated and crack tip running contact is found which results in a region of pure mode II. Applications in general one-dimensional fracture problems also show the validity of the theories. The present Euler beam rule and Timoshenko beam rule are completely analytical rules. They are exact and agree completely with beam FEM predictions. 
The present averaged rule has an excellent agreement with the plane stress FEM predictions. The rule of Hutchinson and Suo [3] is a combined numerical and analytical rule and does not agree with the beam FEM predictions whilst it agrees well with the present averaged rule and plane stress FEM predictions. It does not provide any information on pure mode II region and is not able to deal with shear forces. Moreover, the present method is a completely new approach and provides an excellent tool for studying delamination in fiber reinforced laminated composite beams, plates and shells [20,21].

\section{Acknowledgement}

The authors are very grateful to Professor G. Williams of Imperial College for very helpful discussions on the work.

\section{Appendix}

\section{Fracture mode partition spaces}

By considering the DCB in Figs. 1 or 2 under the tip bending moments $M_{1}$ and $M_{2}$, fracture mode partition spaces in both $\left(M_{1}, M_{2}\right)$ and $\left(M_{1 B}, M_{2 B}\right)$ spaces are studied in this section. Since FEM numerical simulations have a very important role in the study of fracture mechanics of materials and numerical problems can arise in the prediction of the second pure pairs $F D$ $\left(\theta_{E}^{\prime}, \beta_{E}^{\prime}\right)$ and $F D$ - $\left(\theta_{T}^{\prime}, \beta_{T}^{\prime}\right)$ as mentioned above, the numerically generated second 'pure' pair $F D$ $\left(\theta^{\prime}, \beta^{\prime}\right)$ will be considered here in a more useful way to provide some bench mark solutions for interested researchers in FEM simulations. Moreover, the discussion is also useful to experimental studies as a second 'pure' pair $F D-\left(\theta^{\prime}, \beta^{\prime}\right)$ can also be generated experimentally.

Without losing any generality, it is assumed that $\gamma \geq 1$ in the following study. The right half space of $\left(M_{1}, M_{2}\right)$ in Fig. A1 is studied first. The bending moment $M_{1}$ is fixed at a positive value while the bending moment $M_{2}$ varies in $(-\infty, \infty)$, i.e. from A to G. From A to $\mathrm{F}$, $M_{1 B}=M_{1}$ and $M_{2 B}=M_{2}$. As shown in Fig. A2, $\theta$ and $\theta^{\prime}$ mode I occur at $\mathrm{B}$ and $\mathrm{C}$ corresponding to zero crack tip shearing displacement and force, respectively, whilst $\beta$ and $\beta^{\prime}$ mode II occur at $\mathrm{D}$ and $\mathrm{E}$ corresponding to zero crack tip opening force and opening displacement, respectively. From $\mathrm{E}$ to $\mathrm{F}$, crack tip running contact occurs which results in zero relative opening displacement. Thus, pure mode II occurs from $\mathrm{E}$ to $\mathrm{F}$ throughout. From $\mathrm{F}$ to $\mathrm{G}$, DCB tip contact occurs. The contact force can be calculated as

$$
P_{c} a=\frac{3\left(M_{2}-\gamma^{3} M_{1}\right)}{2\left(1+\gamma^{3}\right)}
$$

The crack tip bending moments are therefore given by

$$
M_{1 B}=\frac{3 M_{2}-\left(\gamma^{3}-2\right) M_{1}}{2\left(1+\gamma^{3}\right)}
$$

and

$$
M_{2 B}=\frac{\left(2 \gamma^{3}-1\right) M_{2}+3 \gamma^{3} M_{1}}{2\left(1+\gamma^{3}\right)}
$$

Eqs. (A2) and (A3) give 


$$
M_{2 B}=k M_{1 B}+\left(1+\gamma^{3}\right) M_{1} / 3
$$

where

$$
k=\left(2 \gamma^{3}-1\right) / 3
$$

Since $k$ is always smaller than $\gamma^{3}$, the path at $\mathrm{F}$ will be deflected into the $\left(M_{1 B}, M_{2 B}\right)$ space corresponding to the route from $\mathrm{F}$ to $\mathrm{G}$ in the $\left(M_{1}, M_{2}\right)$ space. Three distinctive paths as shown in Fig. A2 can occur depending on the value of $k$, i.e. $\gamma$. When $k>\beta^{\prime}$, the fracture mode will remain in the pure mode II region. When $\beta<k<\beta^{\prime}$ the path will pass through the $\beta^{\prime}$ mode and enter the mixed mode region. When $k<\beta$, the path will pass through both the $\beta^{\prime}$ and $\beta$ modes. Now, the left half space of $\left(M_{1}, M_{2}\right)$ in Fig. A1 is studied. The bending moment $M_{1}$ is fixed at a negative value while the bending moment $M_{2}$ varies in $(-\infty, \infty)$, i.e. from $\mathrm{H}$ to $\mathrm{L}$. From H to I, $M_{1 B}=M_{1}$ and $M_{2 B}=M_{2}$. From I to L, DCB tip contact occurs. Contact force and crack tip bending moments are given in Eqs. (A1-A3). Again, the path at I will be deflected in the $\left(M_{1 B}, M_{2 B}\right)$ space corresponding to the route from I to $\mathrm{L}$ in the $\left(M_{1}, M_{2}\right)$ space. Three distinctive paths as shown in Fig. A2 can occur depending on the value of $k$, i.e. $\gamma$. All the three paths enter the right half space at J, pass the two pure modes I and enter the upper half space at $\mathrm{K}$. When, $k>\beta^{\prime}$ the path will pass through both $\beta^{\prime}$ and $\beta$ modes and enter the pure mode II region. When $\beta<k<\beta^{\prime}$, the path will pass through the $\beta$ mode. When $k<\beta$, pure mode II does not occur. It is also noted that upper half space above $\gamma^{3}$ is physically prohibited in the $\left(M_{1 B}, M_{2 B}\right)$ space. As mentioned earlier, the interaction between $\theta$ mode I and $\beta$ mode II results in energy flow $\Delta G_{\theta \beta}$ between mode I and mode II. Eqs. (37) and (38) show that in the first quadrant of $\left(\alpha_{\theta}, \alpha_{\beta}\right)$, the flow is from mode II to mode I while in the second and fourth quadrants, the flow is from mode I to mode II. Fig. A3 shows the fracture mode partitions in terms of the variations of $G_{I} / G$ and $G_{I I} / G$. Also, the signs of crack tip opening and shear forces, i.e. $F_{n B}$ and $F_{s B}$, and crack tip relative opening and shearing displacements, i.e. $D_{o p}$ and $D_{s h}$ are shown to help understanding the partitions.

\section{References}

1. Williams JG. On the calculation of energy release rates for cracked laminates. International Journal of Fracture Mechanics 1988;36:101-19.

2. Schapery RA, Davidson BD. Prediction of energy release rate for mixed-mode delamination using classical plate theory. Applied Mechanics Review 1990;43:S281-7.

3. Hutchinson JW, Suo Z. Mixed mode cracking in layered materials. Advances in Applied Mechanics 1992;29:63-191.

4. Suo Z, Hutchinson JW. Interface ceack between two elastic layers. International Journal of Fracture Mechanics 1990;43:1-18.

5. Thouless MD, Evans AG, Ashby MF, Hutchinson JW. The edge cracking and saplling of brittle plates. Acta Metall 1987;35:1333-41.

6. Thouless MD. Fracture of a model interface under mixed mode loading. Acta Metall. Matter 1990;35:1135-40.

7. Wang J, Qiao P. Interface crack between two shear deformable elastic layers. Journal of the Mechanics and Physics of Solids 2004;52:891-905. 
8. Nguyen C, Levy AJ. An exact theory of interfacial debonding in layered elastic composites. International Journal of solids and Structures 2009;46:2712-23.

9. Yan Y, Shang F. Cohesive zone modelling of interfacial delamination in PZT thin films. International Journal of solids and Structures 2009;46:2739-49.

10. Ouyang Z, Li G. Nonlinear interface shear fracture of end notched flexure specimens. International Journal of solids and Structures 2009;46:2659-68.

11. Zou Z, Reid SR, Li S, Soden PD. General expressions for energy release rates for delamination in composite laminates. Proc. R. Soc. Lond. A 2002;458:645-67.

12. Li S, Reid SR, Zou Z. Modelling damage of multiple delaminations and transverse matrix cracking in laminated composites due to low velocity lateral impact. Composite Science and Technology 2005;66:827-36.

13. Hong CS, Shim JY. Strain energy release rate of modified ENF specimen for mixed mode fracture. Journal of Reinforced Plastics and Composites 1993;12:1295-310.

14. Zhang Y. The use of layer-wise theory in the analysis of composite plates. PhD Thesis, 2002, University of Loughborough, England, U.K.

15. Wang S, Harvey C. Fracture mode partition rules for DCB. $17^{\text {th }}$ Interactional Conference on Composite/Nano Engineering (ICCE-17), July 2009, Honolulu, Hawaii, USA.

16. Harvey C, Wang S. Modelling of delamination propagation in composite laminated beam structures. Proceedings of the $7^{\text {th }}$ International Conference of Computational Methods in Science and Engineering (ICCMSE 2009), Simos T E (ed), American Institute of Physics, Rhodes, Greece.

17. Wang S, Harvey C. Mixed mode partition in one dimensional fracture. Journal of Key Engineering Materials 2011;462-63:616-21. (Also, A plenary lecture in the $8^{\text {th }}$ International Conference on Fracture and Strength of Solids (FEOFS 2010), 7-9 ${ }^{\text {th }}$ June 2010, Kuala Lumpur, Malaysia)

18. Wang S, Harvey C. Fracture mode partition theory for one dimensional fracture, presented at the $18^{\text {th }}$ European Conference on Fracture (ECF-18), 30 ${ }^{\text {th }}$ August $-3^{\text {rd }}$ September 2010, Dresden, Germany.

19. Wang S, Guan L. On fracture mode partitions, Computational Material Sciences 2012;52:240-45.

20. Wang S, Harvey C. A theory of one dimensional fracture. Composite Structures 2011 (on line) (Also, A plenary lecture in the $16^{\text {th }}$ International Conference on Composite Structures (ICCS16), 28-30 ${ }^{\text {th }}$ June 2011, Porto, Portugal).

21. Wang S, Harvey C. Partition of mixed modes in Double Cantiliver Beams with non-rigid elastic interfaces and geometrical nonlinearity. $6^{\text {th }}$ International conference on the Fracture of Polymers, Composites and Adhesives 11-15 ${ }^{\text {th }}$ September 2011, les Diablerets, Switzerland.

22. Zhang Y, Wang S. Buckling, post-buckling and delamination propagation in debonded composite laminates Part 1 Theoretical development. Composite Structures 2009;88:121-30 (Also, a plenary lecture in the $16^{\text {th }}$ International Conference on Composite/Nano Engineering (ICCE-16), July 2008, Kunming, China).

23. Wang S, Zhang Y. Buckling, post-buckling and delamination propagation in debonded composite laminates Part 2 Numerical applications. Composite Structures 2009;88:131-46 (Also, a plenary lecture in the $16^{\text {th }}$ International Conference on Composite/Nano Engineering (ICCE-16), July 2008, Kunming, China). 


\section{Table Captions}

Table 1: Energy release rate partitions with shear modulus $G_{x z}=10^{4}$.

Table 2: Energy release rate partitions with shear modulus $G_{x z}=1 / 2.6$.

Table 3: Local and global pureness predictions using $15 \times 2$ Cubic Euler beam elements.

Table 4: Local and global pureness predictions using $15 \times 2$ linear Timoshenko beam elements with $G_{x z}=10^{4}$.

Table 5: Local and global pureness predictions using $820 \times 2$ linear Timoshenko beam elements with $G_{x z}=1 / 2.6$

Table 6: Local and global pureness predictions using 320×6 QUAD4 elements with $G_{x z}=1 / 2.6$.

Table 7: Comparisons between various theories and FEM simulations with varying $M_{2},\left(M_{1}=1\right.$, $\left.N_{1}=0, N_{2}=0, P_{1}=0, P_{2}=0, G_{x z}=1 / 2.6, \gamma=2\right)$.

Table 8: Comparisons between various theories and FEM simulations with varying $M_{2},\left(M_{1}=1\right.$, $\left.N_{1}=0, N_{2}=0, P_{1}=0, P_{2}=0, G_{x z}=1 / 2.6, \gamma=4\right)$.

Table 9: Comparisons between various theories and FEM simulations with varying $N_{1},\left(M_{1}=1\right.$, $\left.M_{2}=0, N_{2}=0, P_{1}=0, P_{2}=0, G_{x z}=1 / 2.6, \gamma=2\right)$.

Table 10: Comparisons between various theories and FEM simulations with varying $N_{2}$, $\left(M_{1}=0, M_{2}=0, N_{1}=1, P_{1}=0, P_{2}=0, G_{x z}=1 / 2.6, \gamma=2\right)$.

Table 11: Through thickness shear effects on energy release rate.

Table 12: Comparisons between various theories and FEM simulations with varying $P_{2}$, $\left(M_{1}=0, M_{2}=0, N_{1}=0, N_{2}=0, P_{1}=1, G_{x z}=1 / 2.6, \gamma=2\right)$.

Table 13: Data of the clamped beam.

Table 14: Energy release rates $\left(\mathrm{J} / \mathrm{m}^{2}\right)$ for $\theta_{1}$ and $\beta_{1}$ modes of a fully clamped beam with a central crack.

Table 15: Data of the clamped axisymmetric plate.

Table 16: Energy release rates $\left(\mu \mathrm{J} / \mathrm{m}^{2}\right)$ for $\theta_{1}$ and $\beta_{1}$ modes of an axisymmetric plate with a central crack. 


\section{Figure Captions}

Fig. 1: A DCB with two tip bending moments.

Fig. 2: A DCB with $\Delta a$ region (a) general description (b) interface stresses of the $\Delta a$ region.

Fig. 3: A clamped beam with a central delamination.

Fig. 4: An axisymmetric plate with a central delamination.

Fig. 5: Energy release rate partitions with shear modulus $G_{x z}=10^{4}$.

Fig. 6: Energy release rate partitions with shear modulus $G_{x z}=1 / 2.6$.

Fig. 7: Comparisons between various theories and FEM simulations with varying $M_{2},\left(M_{1}=1\right.$, $\left.N_{1}=0, N_{2}=0, P_{1}=0, P_{2}=0, G_{x z}=1 / 2.6, \gamma=2\right)$.

Fig. 8: Comparisons between various theories and FEM simulations with varying $M_{2},\left(M_{1}=1\right.$, $\left.N_{1}=0, N_{2}=0, P_{1}=0, P_{2}=0, G_{x z}=1 / 2.6, \gamma=4\right)$.

Fig. 9: Comparisons between various theories and FEM simulations with varying $N_{1},\left(M_{1}=1\right.$, $\left.M_{2}=0, N_{2}=0, P_{1}=0, P_{2}=0, G_{x z}=1 / 2.6, \gamma=2\right)$.

Fig. 10: Comparisons between various theories and FEM simulations with varying $N_{2},\left(M_{1}=0\right.$, $\left.M_{2}=0, N_{1}=1, P_{1}=0, P_{2}=0, G_{x z}=1 / 2.6, \gamma=2\right)$.

Fig. 11: Comparisons between various theories and FEM simulations with varying $P_{2},\left(M_{1}=0\right.$, $\left.M_{2}=0, N_{1}=0, N_{2}=0, P_{1}=1, G_{x z}=1 / 2.6, \gamma=2\right)$.

Fig. A1: Fracture mode partition in $\left(M_{1}, M_{2}\right)$ space.

Fig. A2: Fracture mode partition in $\left(M_{1 B}, M_{2 B}\right)$ space.

Fig. A3: Variations of $G_{I} / G$ and $G_{I I} / G$ in $\left(M_{1}, M_{2}\right)$ space. 
Table 1: Energy release rate partitions with shear modulus $G_{x z}=10^{4}$.

\begin{tabular}{cccc}
\hline & \multicolumn{3}{c}{$G_{I} / G[\%]$} \\
\cline { 2 - 4 }$M_{2}$ & Analytical & $\begin{array}{c}\text { Numerical } \\
\text { (Euler beams) }\end{array}$ & $\begin{array}{c}\text { Numerical } \\
\text { (Timo. beams) }\end{array}$ \\
\hline-10 & 71.43 & 78.57 & 71.43 \\
-8 & 78.35 & 84.23 & 78.35 \\
-6 & 87.85 & 91.62 & 87.85 \\
-4 & 100.00 & 100.00 & 100.00 \\
-2 & 107.59 & 101.14 & 107.59 \\
0 & 76.92 & 65.38 & 76.92 \\
2 & 14.29 & 10.00 & 14.29 \\
4 & -7.14 & -2.86 & -7.14 \\
6 & -5.53 & 2.76 & -5.53 \\
8 & 0.00 & 10.00 & 0.00 \\
10 & 5.13 & 15.91 & 5.13
\end{tabular}

With crack tip running contact and DCB tip contact

\begin{tabular}{cccc}
5.24 & No contact & $\begin{array}{c}\text { Running } \\
\text { contact starts }\end{array}$ & No contact \\
6 & No contact & 0 & No contact \\
8 & DCB tip & DCB tip & DCB tip \\
10 & contact starts & contact starts & contact starts \\
\hline
\end{tabular}


Table 2: Energy release rate partitions with shear modulus $G_{x z}=1 / 2.6$.

\begin{tabular}{cccc}
\hline & \multicolumn{3}{c}{$G_{I} / G[\%]$} \\
\cline { 2 - 4 }$M_{2}$ & $\begin{array}{c}\text { Analytical } \\
(\text { Timoshenko) }\end{array}$ & $\begin{array}{c}\text { Numerical } \\
(201 \times 2 \text { Timo. } \\
\text { beams })\end{array}$ & $\begin{array}{c}\text { Numerical } \\
(801 \times 2 \text { Timo. } \\
\text { beams })\end{array}$ \\
\hline-10 & 89.29 & 84.48 & 87.78 \\
-8 & 93.04 & 89.09 & 91.80 \\
-6 & 97.27 & 94.74 & 96.47 \\
-4 & 100.00 & 100.00 & 100.00 \\
-2 & 91.46 & 95.79 & 92.81 \\
0 & 48.08 & 55.83 & 50.51 \\
2 & 3.57 & 6.45 & 4.47 \\
4 & 3.57 & 0.69 & 2.67 \\
6 & 15.20 & 9.63 & 13.46 \\
8 & 25.00 & 18.28 & 22.90 \\
10 & 32.08 & 24.84 & 29.82
\end{tabular}

With crack tip running contact and DCB tip contact

$20 / 7$

3.12

3.76

8

10
Running contact starts

No contact

No contact

Running contact starts

DCB tip contact starts
No contact

Running contact starts

0

DCB tip contact starts 0 
Table 3: Local and global pureness predictions using $15 \times 2$ Cubic Euler beam elements.

\begin{tabular}{cccccc}
\hline \multicolumn{5}{c}{$G_{I} / G[\%]$ for $n$ spring pairs } \\
\cline { 2 - 6 }$M_{2}$ & 1 & 2 & 3 & 4 & 5 \\
\hline-1 & 90.00 & 96.00 & 97.20 & 97.92 & 98.33 \\
8 & 10.00 & 4.00 & 2.80 & 2.08 & 1.67 \\
-4 & 100.00 & 100.00 & 100.00 & 100.00 & 100.00 \\
$20 / 7$ & 0.00 & 0.00 & 0.00 & 0.00 & 0.00 \\
\hline
\end{tabular}


Table 4: Local and global pureness predictions using $15 \times 2$ linear Timoshenko beam elements with $G_{x z}=10^{4}$.

\begin{tabular}{cccccc}
\hline & \multicolumn{5}{c}{$G_{I} / G[\%]$ for $n$ spring pairs } \\
\cline { 2 - 6 }$M_{2}[\mathrm{~N}]$ & 1 & 2 & 3 & 4 & 5 \\
\hline-1 & 100.00 & 100.00 & 100.00 & 100.00 & 100.00 \\
8 & 0.00 & 0.00 & 0.00 & 0.00 & 0.00 \\
-4 & 100.00 & 100.00 & 100.00 & 100.00 & 100.00 \\
$20 / 7$ & 0.00 & 0.00 & 0.00 & 0.00 & 0.00 \\
\hline
\end{tabular}


Table 5: Local and global pureness predictions using $820 \times 2$ linear Timoshenko beam elements with $G_{x z}=1 / 2.6$.

\begin{tabular}{cccccccc}
\hline & \multicolumn{7}{c}{$G_{I} / G[\%]$ for $n$ spring pairs } \\
\cline { 2 - 8 }$M_{2}[\mathrm{~N}]$ & 1 & 2 & 3 & 4 & 5 & 10 & 20 \\
\hline-1 & 77.10 & 79.03 & 80.74 & 82.27 & 83.62 & 88.55 & 93.37 \\
8 & 22.90 & 20.97 & 19.26 & 17.73 & 16.38 & 11.45 & 6.63 \\
-4 & 100.00 & 100.00 & 100.00 & 100.00 & 100.00 & 100.00 & 100.00 \\
$20 / 7$ & 0.00 & 0.00 & 0.00 & 0.00 & 0.00 & 0.00 & 0.00 \\
\hline
\end{tabular}


Table 6: Local and global pureness predictions using 320×6 QUAD4 elements with $G_{x z}=1 / 2.6$.

\begin{tabular}{cccccccc}
\hline & \multicolumn{7}{c}{$G_{I} / G[\%]$ for $n$ spring pairs } \\
\cline { 2 - 8 }$M_{2}$ & 1 & 2 & 3 & 4 & 5 & 10 & 20 \\
\hline-1 & 85.27 & 86.16 & 87.41 & 88.82 & 90.20 & 94.70 & 97.67 \\
8 & 16.10 & 15.19 & 13.91 & 12.47 & 11.06 & 6.47 & 4.25 \\
-4 & 98.43 & 98.39 & 98.34 & 98.31 & 98.31 & 98.49 & 98.20 \\
$20 / 7$ & 1.04 & 1.03 & 1.01 & 0.97 & 0.90 & 0.49 & 0.10 \\
\hline
\end{tabular}


Table 7: Comparisons between various theories and FEM simulations with varying $M_{2},\left(M_{1}=1\right.$, $\left.N_{1}=0, N_{2}=0, P_{1}=0, P_{2}=0, G_{x z}=1 / 2.6, \gamma=2\right)$.

\begin{tabular}{cccccc}
\hline & \multicolumn{5}{c}{$G_{I} / G[\%]$} \\
$M_{2}$ & $\begin{array}{c}\text { Analytical } \\
\text { (Euler) }\end{array}$ & $\begin{array}{c}\text { Analytical } \\
\text { (Timo.) }\end{array}$ & $\begin{array}{c}\text { Averaged } \\
\text { Analytical } \\
\text { (Euler \& } \\
\text { Timo.) }\end{array}$ & $\begin{array}{c}\text { Hutchinson } \\
\text { \& Suo [3] }\end{array}$ & $\begin{array}{c}\text { 2D FEM } \\
(320 \times 6 \\
\text { QUAD4s) }\end{array}$ \\
\hline-10 & 71.43 & 89.29 & 80.36 & 81.05 & 80.48 \\
-8 & 78.35 & 93.04 & 85.70 & 85.97 & 85.39 \\
-6 & 87.85 & 97.27 & 92.56 & 92.20 & 91.69 \\
-4 & 100.00 & 100.00 & 100.00 & 98.64 & 98.43 \\
-2 & 107.59 & 91.46 & 99.53 & 96.81 & 97.39 \\
0 & 76.92 & 48.08 & 62.50 & 59.71 & 60.32 \\
2 & 14.29 & 3.57 & 8.93 & 9.14 & 8.64 \\
4 & -7.14 & 3.57 & -1.79 & 0.53 & 0.57 \\
6 & -5.53 & 15.20 & 4.84 & 7.83 & 8.21 \\
8 & 0.00 & 25.00 & 12.50 & 15.64 & 16.10 \\
10 & 5.13 & 32.08 & 18.61 & 21.75 & 22.19
\end{tabular}

With crack tip running contact and DCB tip contact

\begin{tabular}{|c|c|c|c|c|c|}
\hline $20 / 7$ & No contact & $\begin{array}{c}\text { Running } \\
\text { contact starts }\end{array}$ & No contact & - & No contact \\
\hline $44 / 13$ & No contact & 0 & $\begin{array}{c}\text { Running } \\
\text { contact starts }\end{array}$ & - & No contact \\
\hline 3.67 & No contact & 0 & 0 & - & $\begin{array}{c}\text { Running } \\
\text { contact starts }\end{array}$ \\
\hline 8 & $\begin{array}{c}\text { DCB tip } \\
\text { contact starts }\end{array}$ & $\begin{array}{c}\text { DCB tip } \\
\text { contact starts }\end{array}$ & $\begin{array}{c}\text { DCB tip } \\
\text { contact starts }\end{array}$ & - & $\begin{array}{c}\text { DCB tip } \\
\text { contact starts }\end{array}$ \\
\hline 10 & -2.11 & 0 & 0 & - & 0 \\
\hline
\end{tabular}


Table 8: Comparisons between various theories and FEM simulations with varying $M_{2},\left(M_{1}=1\right.$, $\left.N_{1}=0, N_{2}=0, P_{1}=0, P_{2}=0, G_{x z}=1 / 2.6, \gamma=4\right)$.

\begin{tabular}{cccccc}
\hline & \multicolumn{5}{c}{$G_{I} / G[\%]$} \\
\cline { 2 - 6 }$M_{2}[\mathrm{~N}]$ & $\begin{array}{c}\text { Analytical } \\
\text { (Euler) }\end{array}$ & $\begin{array}{c}\text { Analytical } \\
\text { (Timo.) }\end{array}$ & $\begin{array}{c}\text { Averaged } \\
\text { Analytical } \\
\text { (Euler \& } \\
\text { Timo.) }\end{array}$ & $\begin{array}{c}\text { Hutchinson } \\
\text { \& Suo [3] }\end{array}$ & $\begin{array}{c}\text { 2D FEM } \\
(320 \times 6 \\
\text { QUAD4s) }\end{array}$ \\
\hline-20 & 89.55 & 99.14 & 94.34 & 90.73 & 90.48 \\
-16 & 100.00 & 100.00 & 100.00 & 95.39 & 95.29 \\
-12 & 111.57 & 98.36 & 104.96 & 99.23 & 99.53 \\
-8 & 120.90 & 90.67 & 105.78 & 99.12 & 100.09 \\
-4 & 118.34 & 71.35 & 94.84 & 88.35 & 89.63 \\
0 & 90.32 & 39.52 & 64.92 & 60.99 & 60.70 \\
4 & 42.86 & 10.71 & 26.79 & 27.31 & 24.81 \\
8 & 4.14 & 0.15 & 2.14 & 6.36 & 4.09 \\
12 & -15.07 & 3.19 & -5.94 & 0.14 & -0.67 \\
16 & -21.43 & 10.71 & -5.36 & 1.36 & 1.76 \\
20 & -21.87 & 18.39 & -1.74 & 5.05 & 6.17
\end{tabular}

With crack tip running contact and DCB tip contact

\begin{tabular}{|c|c|c|c|c|c|}
\hline $112 / 13$ & No contact & $\begin{array}{c}\text { Running } \\
\text { contact starts }\end{array}$ & No contact & - & No contact \\
\hline 13.88 & No contact & 0 & No contact & - & $\begin{array}{c}\text { Running } \\
\text { contact starts }\end{array}$ \\
\hline $1104 / 51$ & No contact & 0 & $\begin{array}{c}\text { Running } \\
\text { contact starts }\end{array}$ & - & 0 \\
\hline 64 & $\begin{array}{c}\text { DCB tip } \\
\text { contact starts }\end{array}$ & $\begin{array}{c}\text { DCB tip } \\
\text { contact starts }\end{array}$ & $\begin{array}{l}\text { DCB tip } \\
\text { contact starts }\end{array}$ & - & $\begin{array}{c}\text { DCB tip } \\
\text { contact starts }\end{array}$ \\
\hline 100 & -11.35 & 0 & 0 & - & 0 \\
\hline
\end{tabular}


Table 9: Comparisons between various theories and FEM simulations with varying $N_{1},\left(M_{1}=1\right.$, $\left.M_{2}=0, N_{2}=0, P_{1}=0, P_{2}=0, G_{x z}=1 / 2.6, \gamma=2\right)$.

\begin{tabular}{|c|c|c|c|c|}
\hline \multirow[b]{2}{*}{$N_{1}[\mathrm{~N}]$} & \multicolumn{4}{|c|}{$G_{I} / G[\%]$} \\
\hline & $\begin{array}{l}\text { Analytical } \\
\text { (Euler) }\end{array}$ & $\begin{array}{c}\text { Analytical } \\
\text { (Timo.) }\end{array}$ & $\begin{array}{c}\text { Averaged } \\
\text { Analytical } \\
\text { (Euler \& Timo.) }\end{array}$ & $\begin{array}{c}\text { 2D FEM } \\
(320 \times 6 \\
\text { QUAD4s }) \\
\end{array}$ \\
\hline-10 & 71.43 & 89.29 & 80.36 & 78.26 \\
\hline-8 & 85.71 & 96.43 & 91.07 & 88.74 \\
\hline-6 & 100.00 & 100.00 & 100.00 & 97.82 \\
\hline-4 & 107.69 & 94.23 & 100.96 & 99.19 \\
\hline-2 & 100.00 & 75.00 & 87.50 & 85.74 \\
\hline 0 & 76.92 & 48.08 & 62.50 & 60.32 \\
\hline 2 & 50.00 & 25.00 & 37.50 & 35.46 \\
\hline 4 & 28.57 & 10.71 & 19.64 & 18.41 \\
\hline 6 & 14.29 & 3.57 & 8.93 & 8.65 \\
\hline 8 & 5.41 & 0.68 & 3.04 & 3.57 \\
\hline 10 & 0.00 & 0.00 & 0.00 & 1.13 \\
\hline 12 & -3.28 & 0.41 & -1.43 & 0.12 \\
\hline 14 & -5.26 & 1.32 & -1.97 & -0.12 \\
\hline
\end{tabular}


Table 10: Comparisons between various theories and FEM simulations with varying $N_{2}$, $\left(M_{1}=0, M_{2}=0, N_{1}=1, P_{1}=0, P_{2}=0, G_{x z}=1 / 2.6, \gamma=2\right)$.

\begin{tabular}{ccccc}
\hline & \multicolumn{4}{c}{$G_{I} / G[\%]$} \\
\cline { 2 - 5 }$N_{2}[\mathrm{~N}]$ & $\begin{array}{c}\text { Analytical } \\
\text { (Euler) }\end{array}$ & $\begin{array}{c}\text { Analytical } \\
\text { (Timoshenko) }\end{array}$ & $\begin{array}{c}\text { Averaged } \\
\text { Analytical } \\
\text { (Euler \& Timo.) }\end{array}$ & $\begin{array}{c}\text { 2D FEM } \\
(320 \times 6 \\
\text { QUAD4s) }\end{array}$ \\
\hline-10 & 0 & 25 & 12.5 & 14.72 \\
-8 & 0 & 25 & 12.5 & 14.72 \\
-6 & 0 & 25 & 12.5 & 14.72 \\
-4 & 0 & 25 & 12.5 & 14.72 \\
-2 & 0 & 25 & 12.5 & 14.72 \\
0 & 0 & 25 & 12.5 & 14.72 \\
2 & - & - & - & - \\
4 & 0 & 25 & 12.5 & 14.72 \\
6 & 0 & 25 & 12.5 & 14.72 \\
8 & 0 & 25 & 12.5 & 14.72 \\
10 & 0 & 25 & 12.5 & 14.72 \\
\hline
\end{tabular}


Table 11: Through thickness shear effects on energy release rate.

\begin{tabular}{|c|c|c|c|c|c|c|}
\hline & \multicolumn{3}{|c|}{$G_{x z}=1 / 2.6$} & \multicolumn{3}{|c|}{$G_{x z}=1 / 26$} \\
\hline & Analytical & $\begin{array}{l}\text { Timo- } \\
\text { Beam } \\
\text { FEM }\end{array}$ & $\begin{array}{c}\text { 2D FEM } \\
(330 \times 6 \\
\text { QUAD4s })\end{array}$ & Analytical & $\begin{array}{l}\text { Timo- } \\
\text { Beam } \\
\text { FEM }\end{array}$ & $\begin{array}{c}\text { 2D FEM } \\
(330 \times 6 \\
\text { QUAD4s) }\end{array}$ \\
\hline$G_{I}$ & 1854.1 & 1824.9 & 1788.1 & 2467.6 & 2361.9 & 2280.4 \\
\hline$G_{I I}$ & 0 & 0 & 29.1 & 0 & 0 & 37.3 \\
\hline$G$ & 1854.1 & 1824.9 & 1817.2 & 2467.6 & 2361.9 & 2317.7 \\
\hline
\end{tabular}


Table 12: Comparisons between various theories and FEM simulations with varying $P_{2}$, $\left(M_{1}=0, M_{2}=0, N_{1}=0, N_{2}=0, P_{1}=1, G_{x z}=1 / 2.6, \gamma=2\right)$.

\begin{tabular}{ccccc}
\hline & \multicolumn{4}{c}{$G_{I} / G[\%]$} \\
\cline { 2 - 5 }$P_{2}[\mathrm{~N}]$ & $\begin{array}{c}\text { Analytical } \\
\text { (Timoshenko) }\end{array}$ & $\begin{array}{c}\text { Numerical } \\
(830 \times 2 \text { Timo. } \\
\text { beams) }\end{array}$ & $\begin{array}{c}\text { Averaged } \\
\text { Analytical } \\
(\text { Euler \& Timo. })\end{array}$ & $\begin{array}{c}\text { 2D FEM } \\
(330 \times 6 \\
\text { QUAD4s })\end{array}$ \\
\hline-10 & 90.70 & 89.05 & 82.94 & 82.04 \\
-8 & 93.98 & 92.68 & 87.62 & 86.51 \\
-6 & 97.64 & 96.85 & 93.58 & 92.23 \\
-4 & 100.00 & 100.00 & 100.00 & 98.40 \\
-2 & 92.48 & 93.71 & 99.58 & 97.99 \\
0 & 51.05 & 53.79 & 64.64 & 63.61 \\
2 & 3.57 & 5.01 & 8.93 & 9.17 \\
4 & 4.69 & 3.10 & -0.60 & 0.90 \\
6 & 18.12 & 15.15 & 8.12 & 9.87 \\
8 & 28.81 & 25.38 & 16.95 & 18.60 \\
10 & 36.28 & 32.70 & 23.64 & 25.14
\end{tabular}

With crack tip running contact and DCB tip contact

$\begin{array}{ccccc}20 / 7 & \begin{array}{c}\text { Running } \\ \text { contact starts }\end{array} & \text { No contact } & - & \text { No contact } \\ 2.87 & 0 & \text { No contact } & \begin{array}{c}\text { Running } \\ \text { contact starts }\end{array} & \text { No contact } \\ 3.10 & 0 & \begin{array}{c}\text { Running } \\ \text { contact starts }\end{array} & 0 & \text { No contact } \\ 3.58 & 0 & 0 & 0 & \begin{array}{c}\text { Running } \\ \text { contact starts } \\ \text { DCB tip }\end{array} \\ 8 & \begin{array}{c}\text { DCB tip } \\ \text { contact starts }\end{array} & \begin{array}{c}\text { DCB tip } \\ \text { contact starts }\end{array} & \begin{array}{c}\text { DCB tip } \\ \text { contact starts }\end{array} & \begin{array}{c}\text { contact starts } \\ 10\end{array} \\ 0 & 0 & 0 & 0\end{array}$


Table 13: Data of the clamped beam.

\begin{tabular}{ccccccccc}
\hline$E$ & $L$ & $a$ & $b$ & $h_{1}$ & $h_{2}$ & $\gamma$ & $\beta$ & $\theta$ \\
\hline $124 \mathrm{GPa}$ & $25 \mathrm{~mm}$ & $25 \mathrm{~mm}$ & $5 \mathrm{~mm}$ & $1 \mathrm{~mm}$ & $2 \mathrm{~mm}$ & 2 & $20 / 7$ & -4 \\
\hline
\end{tabular}


Table 14: Energy release rates $\left(\mathrm{J} / \mathrm{m}^{2}\right)$ for $\theta_{1}$ and $\beta_{1}$ modes of a fully clamped beam with a central crack.

\begin{tabular}{|c|c|c|c|c|c|c|c|c|c|}
\hline & \multicolumn{3}{|c|}{40 elements } & \multicolumn{3}{|c|}{200 elements } & \multicolumn{3}{|c|}{ Analytical } \\
\hline & $G_{I}$ & $G_{I I}$ & $G$ & $G_{I}$ & $G_{I I}$ & $G$ & $G_{I}$ & $G_{I I}$ & $G$ \\
\hline$\theta_{1}$ & 0.3992 & 0 & 0.3992 & 0.4031 & 0 & 0.4031 & 0.4032 & 0 & 0.4032 \\
\hline$\beta_{1}$ & 0 & 0.2200 & 0.2200 & 0 & 0.2221 & 0.2221 & 0 & 0.2222 & 0.2222 \\
\hline
\end{tabular}


Table 15: Data of the clamped axisymmetric plate.

\begin{tabular}{ccccccccc}
\hline$E$ & $v$ & $R$ & $a$ & $h_{1}$ & $h_{2}$ & $\gamma$ & $\beta$ & $\theta$ \\
\hline $140 \mathrm{GPa}$ & 0.3 & $100 \mathrm{~mm}$ & $20 \mathrm{~mm}$ & $1 \mathrm{~mm}$ & $2 \mathrm{~mm}$ & 2 & $20 / 7$ & -4 \\
\hline
\end{tabular}


Table 16: Energy release rates $\left(\mu \mathrm{J} / \mathrm{m}^{2}\right)$ for $\theta_{1}$ and $\beta_{1}$ modes of an axisymmetric plate with a central crack.

\begin{tabular}{|c|c|c|c|c|c|c|c|c|c|}
\hline & \multicolumn{3}{|c|}{40 elements } & \multicolumn{3}{|c|}{200 elements } & \multicolumn{3}{|c|}{ Analytical } \\
\hline & $G_{I}$ & $G_{I I}$ & $G$ & $G_{I}$ & $G_{I I}$ & $G$ & $G_{I}$ & $G_{I I}$ & $G$ \\
\hline$\theta_{1}$ & 740.9 & 0 & 740.9 & 675.2 & 0 & 675.2 & 658.6 & 0 & 658.6 \\
\hline$\beta_{1}$ & 0 & 408.3 & 408.3 & 0 & 372.0 & 372.0 & 0 & 362.9 & 362.9 \\
\hline
\end{tabular}




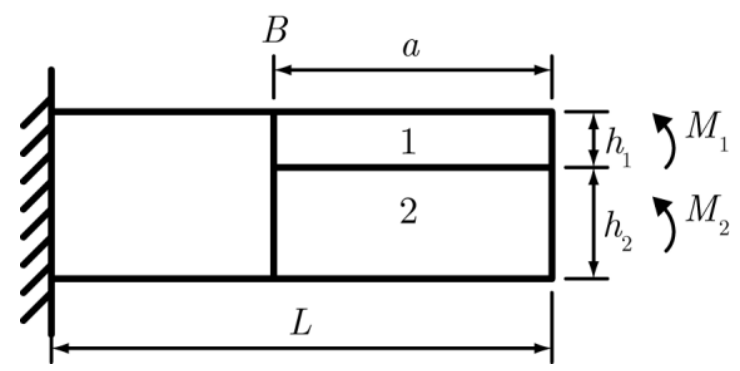

Fig. 1: A DCB with two tip bending moments. 


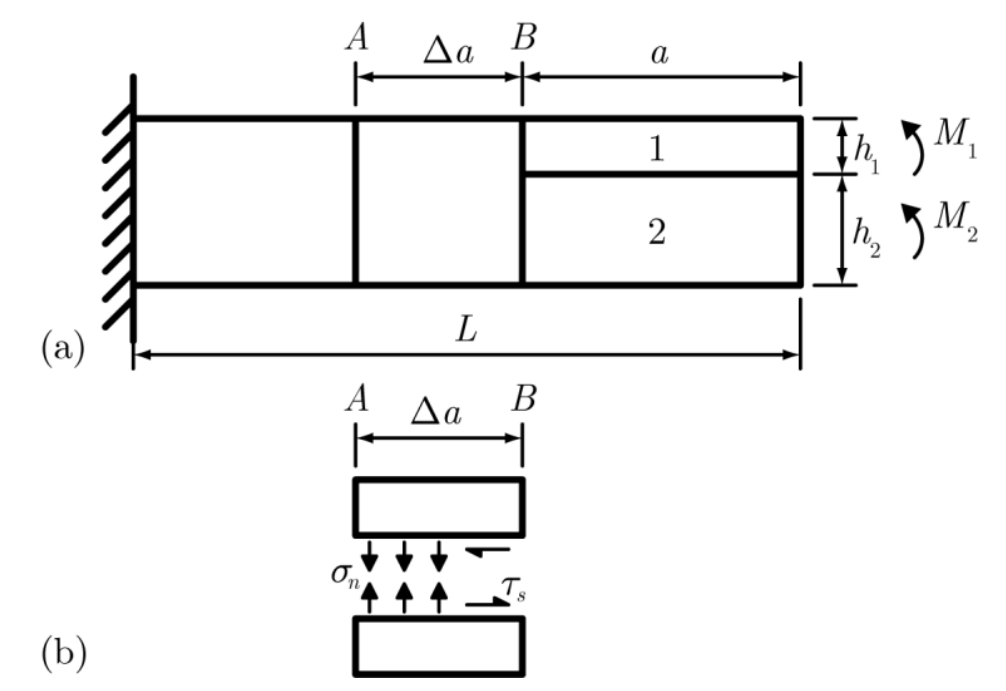

Fig. 2: A DCB with $\Delta a$ region (a) general description (b) interface stresses of the $\Delta a$ region. 


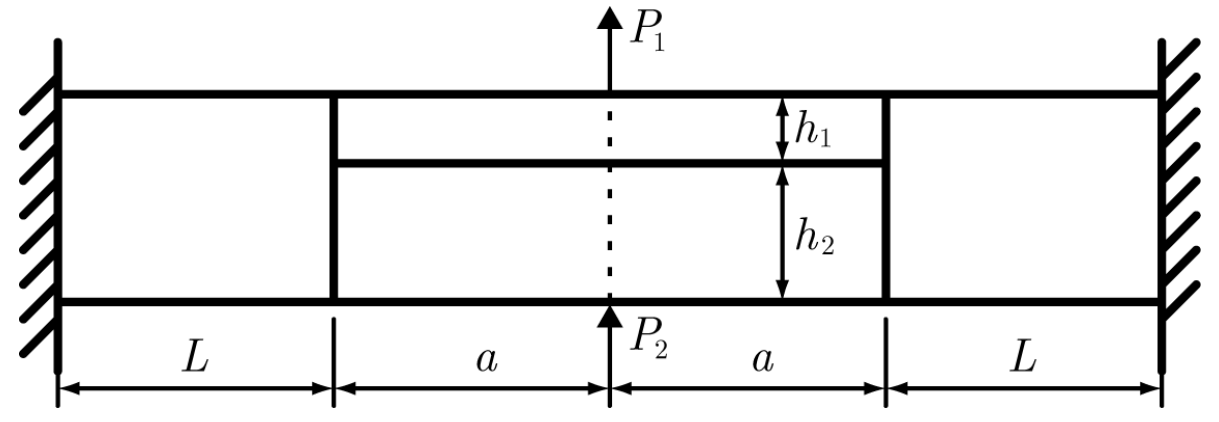

Fig. 3: A clamped beam with a central delamination. 


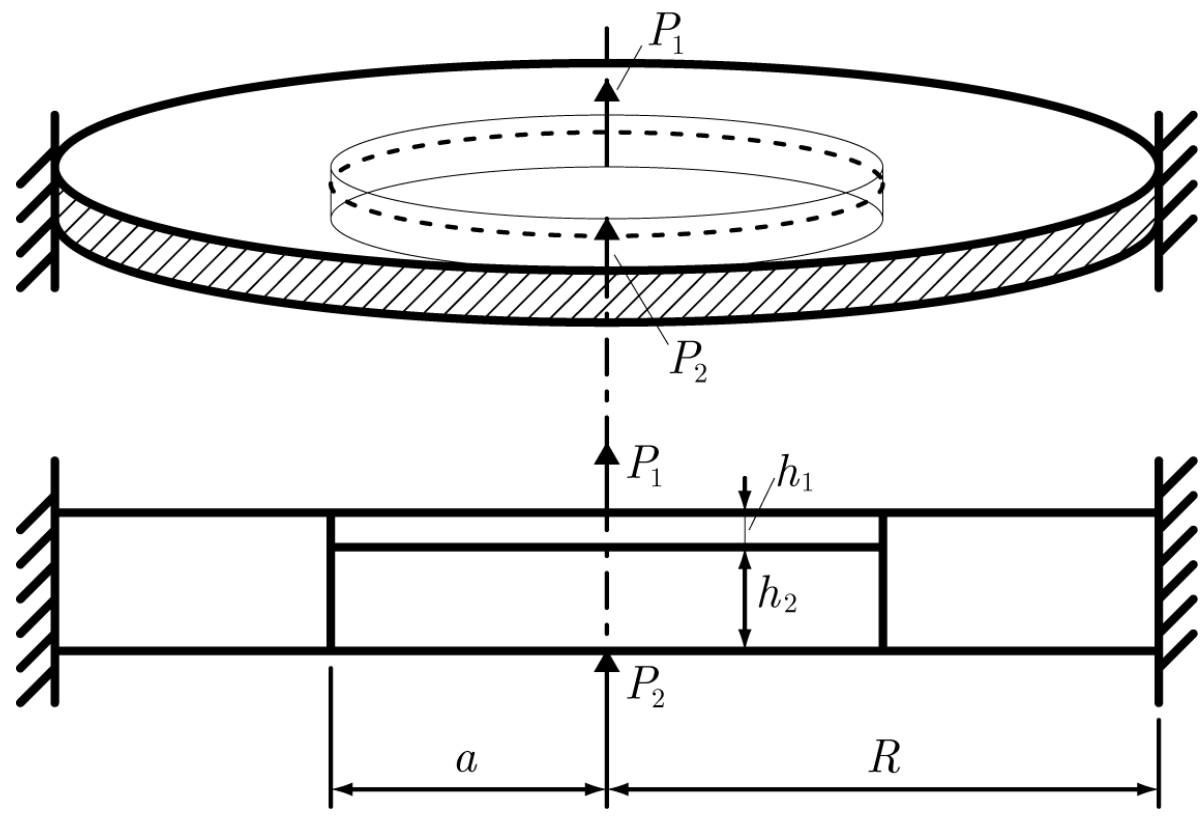

Fig. 4: An axisymmetric plate with a central delamination. 


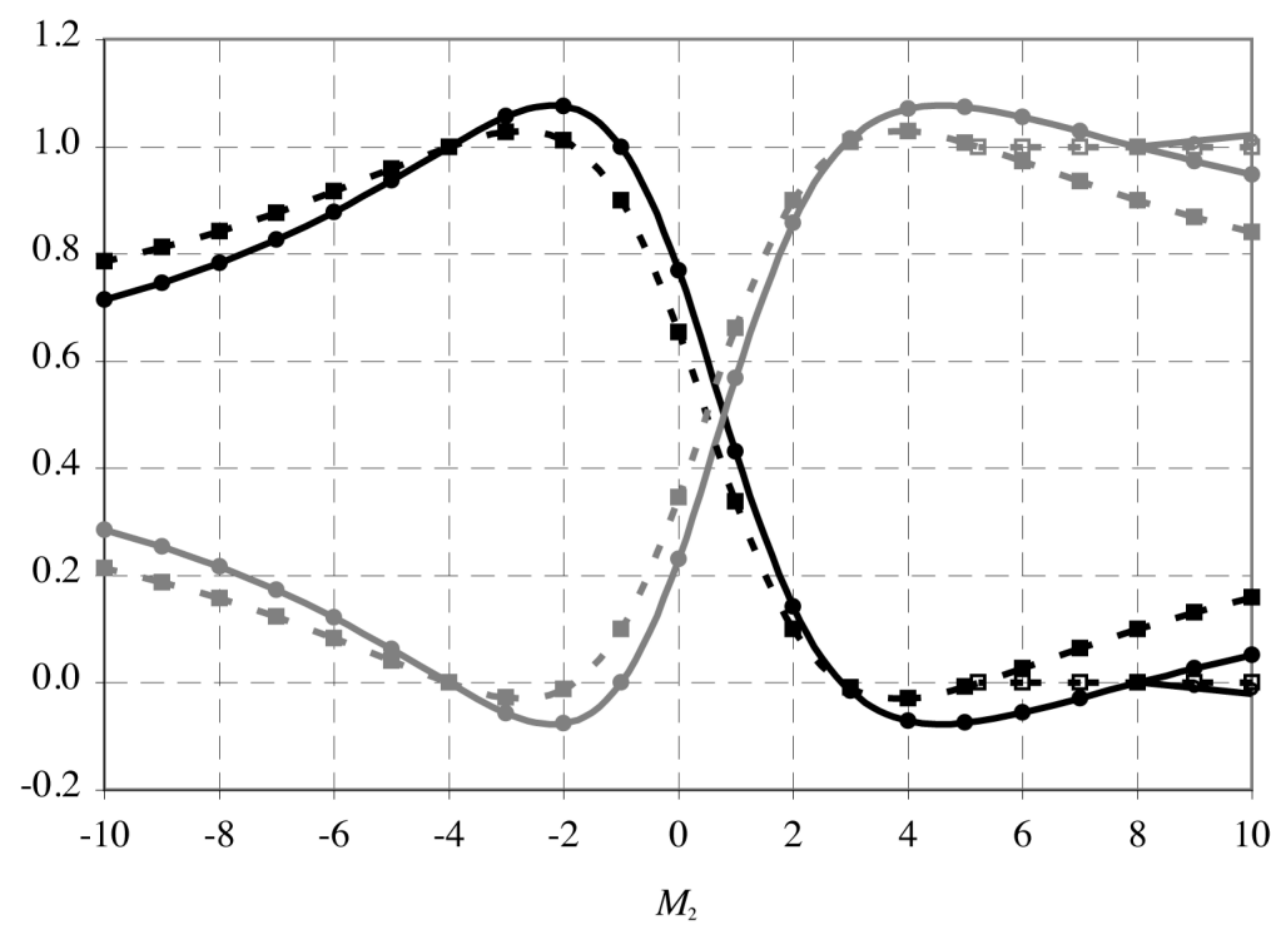

Analytical Euler $G_{\mathrm{I}} / G$

Analytical Euler $G_{\mathrm{II}} / G$

- - - ' $11 \times 2$ Numerical Euler $G_{\mathrm{I}} / G \quad$ - $=$ - $11 \times 2$ Numerical Euler $G_{\mathrm{II}} / G$

- $11 \times 2$ Numerical Timo. $G_{\mathrm{I}} / G \quad \bullet \quad 11 \times 2$ Numerical Timo. $G_{\mathrm{II}} / G$

Fig. 5: Energy release rate partitions with shear modulus $G_{x z}=10^{4}$. 


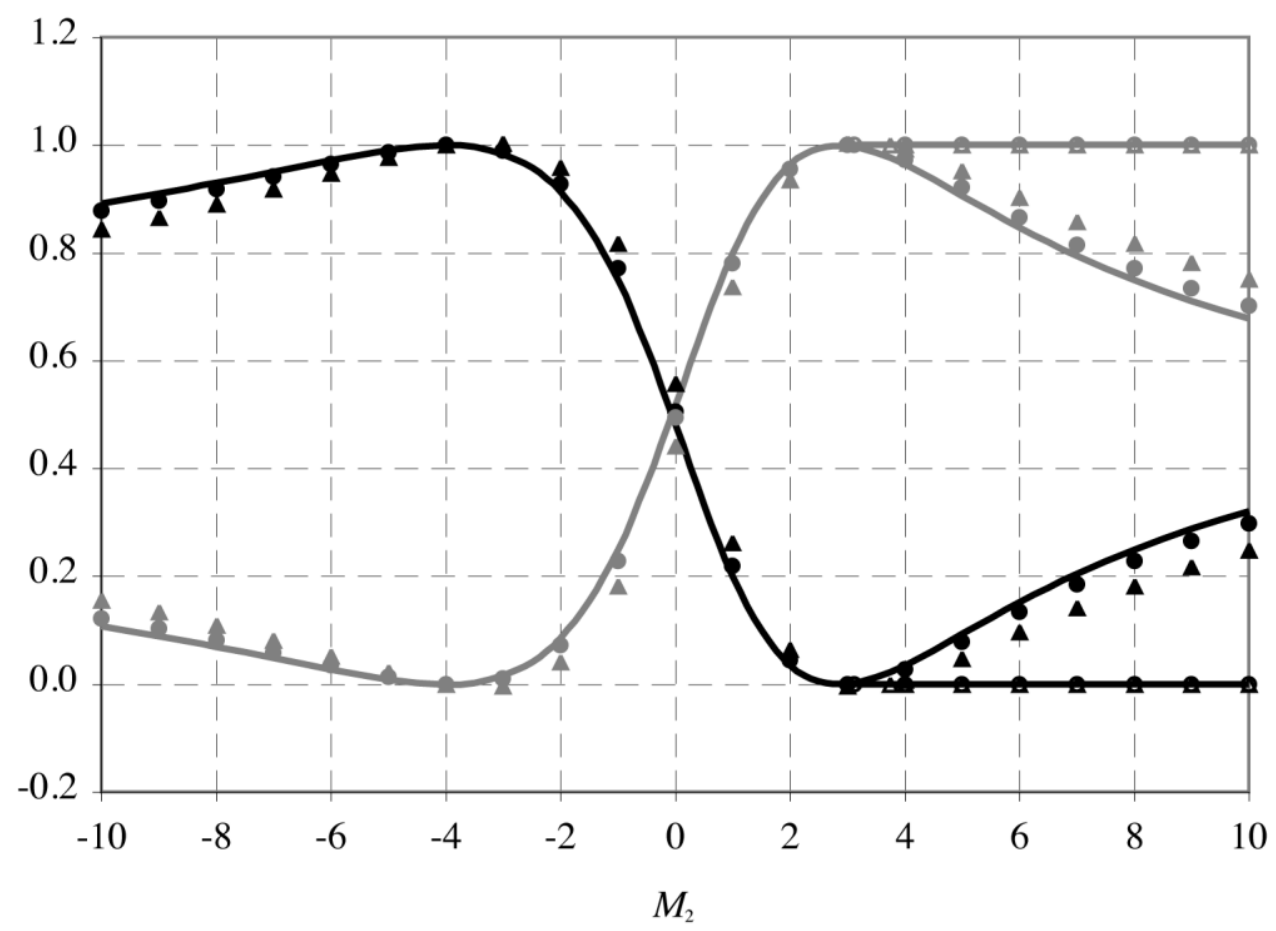
- Analytical Timo. $G_{\mathrm{I}} / G$
Analytical Timo. $G_{\mathrm{II}} / G$
- $801 \times 2$ Numerical Timo. $G_{\mathrm{I}} / G$
- $801 \times 2$ Numerical Timo. $G_{\mathrm{II}} / G$
- $201 \times 2$ Numerical Timo. $G_{\mathrm{I}} / G$
- $201 \times 2$ Numerical Timo. $G_{\mathrm{II}} / G$

Fig. 6: Energy release rate partitions with shear modulus $G_{x z}=1 / 2.6$. 

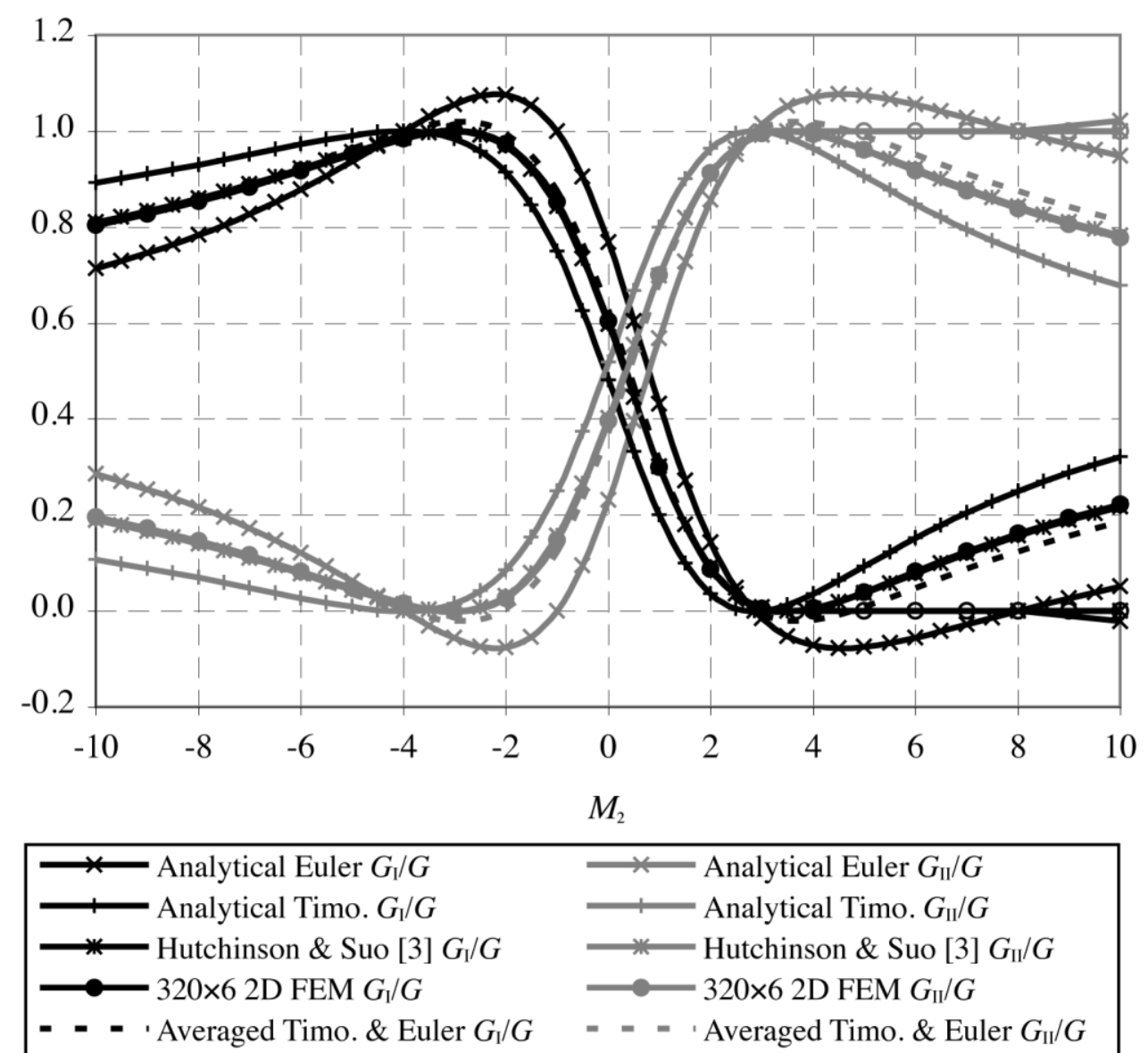

Fig. 7: Comparisons between various theories and FEM simulations with varying $M_{2},\left(M_{1}=1\right.$, $\left.N_{1}=0, N_{2}=0, P_{1}=0, P_{2}=0, G_{x z}=1 / 2.6, \gamma=2\right)$. 

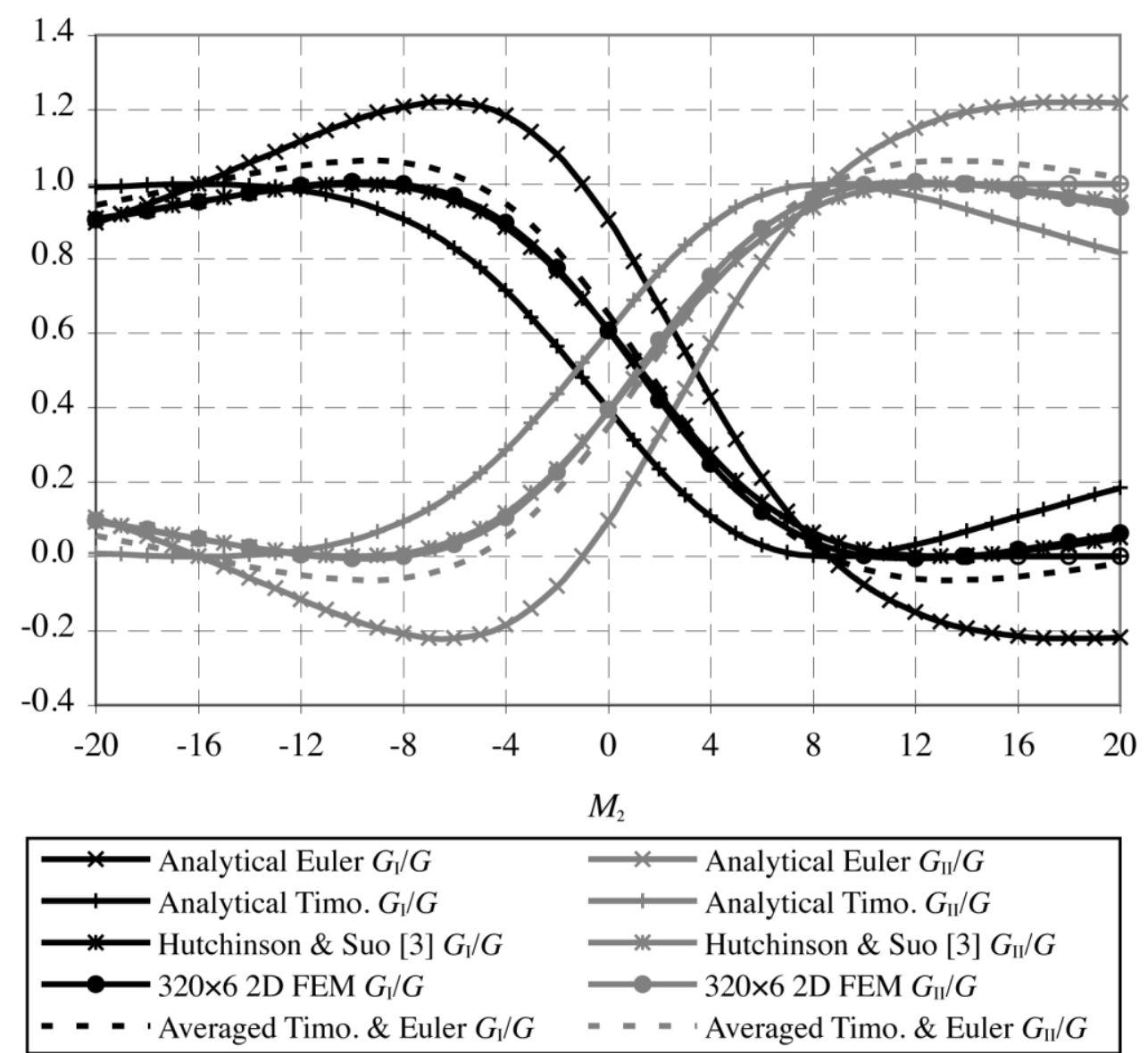

Fig. 8: Comparisons between various theories and FEM simulations with varying $M_{2},\left(M_{1}=1\right.$, $\left.N_{1}=0, N_{2}=0, P_{1}=0, P_{2}=0, G_{x z}=1 / 2.6, \gamma=4\right)$. 


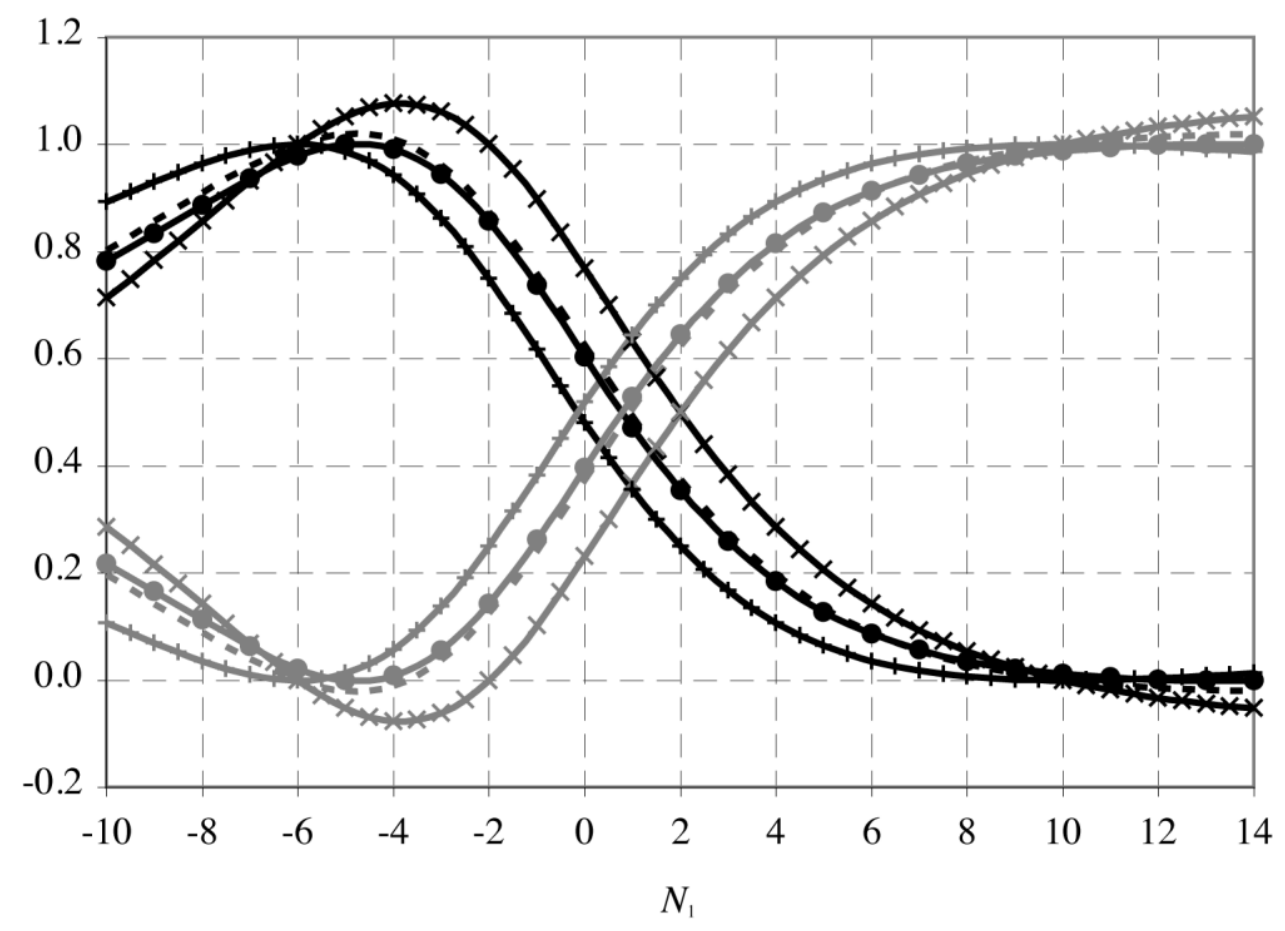

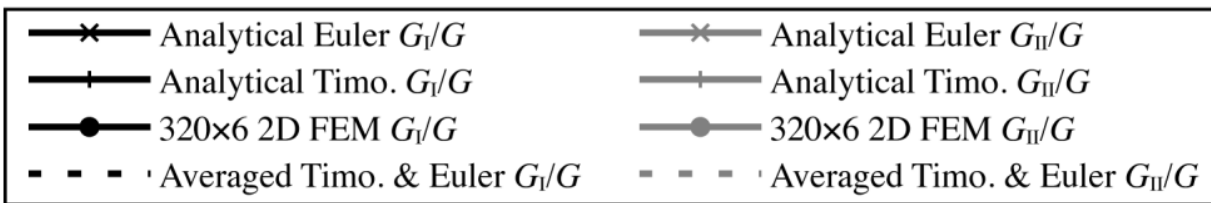

Fig. 9: Comparisons between various theories and FEM simulations with varying $N_{1},\left(M_{1}=1\right.$, $\left.M_{2}=0, N_{2}=0, P_{1}=0, P_{2}=0, G_{x z}=1 / 2.6, \gamma=2\right)$. 


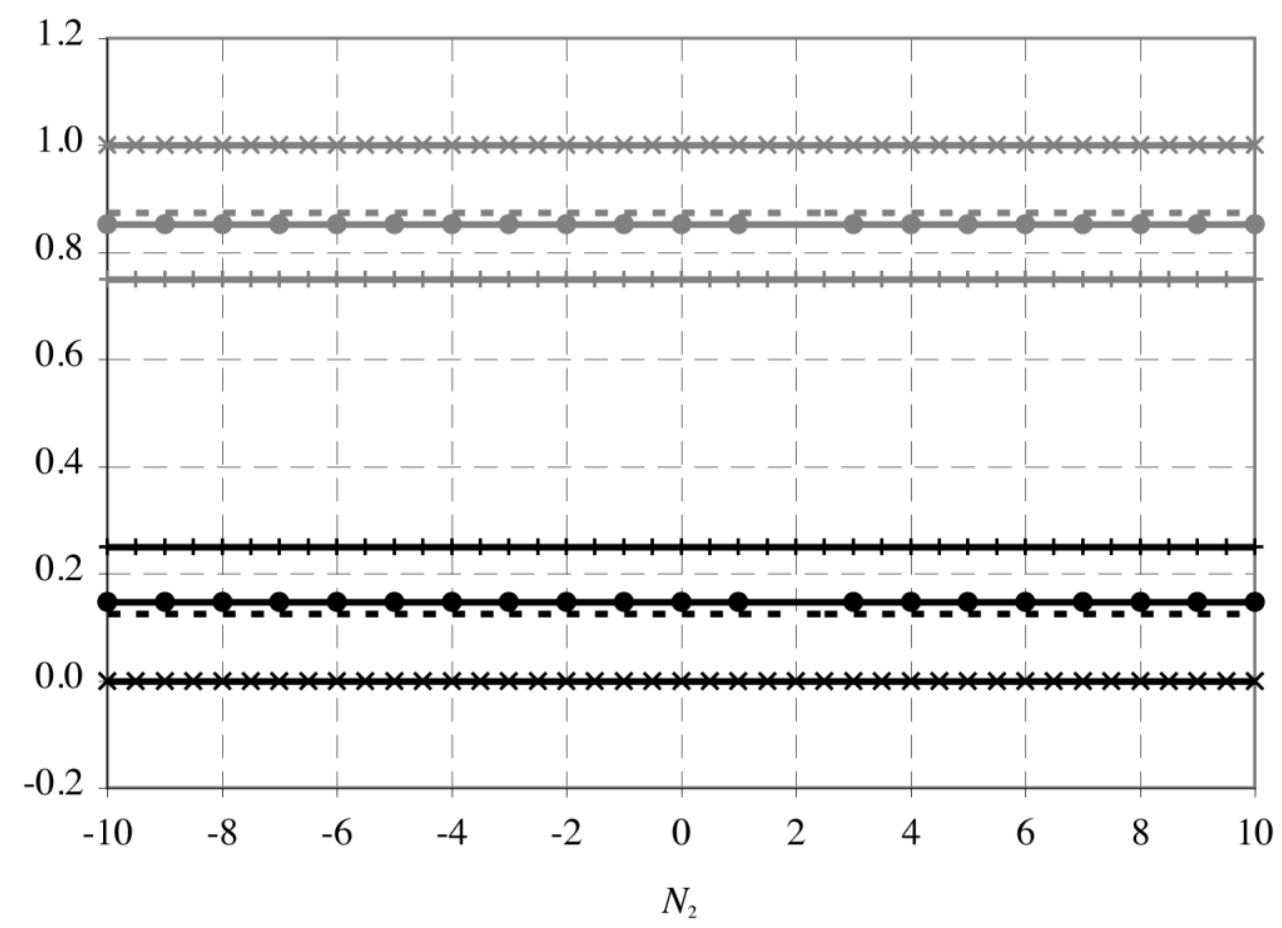

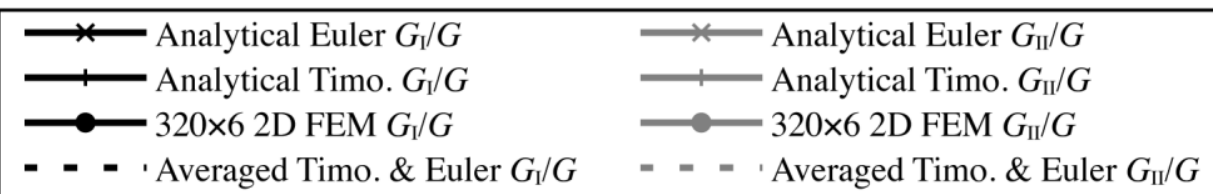

Fig. 10: Comparisons between various theories and FEM simulations with varying $N_{2},\left(M_{1}=0\right.$, $\left.M_{2}=0, N_{1}=1, P_{1}=0, P_{2}=0, G_{x z}=1 / 2.6, \gamma=2\right)$. 


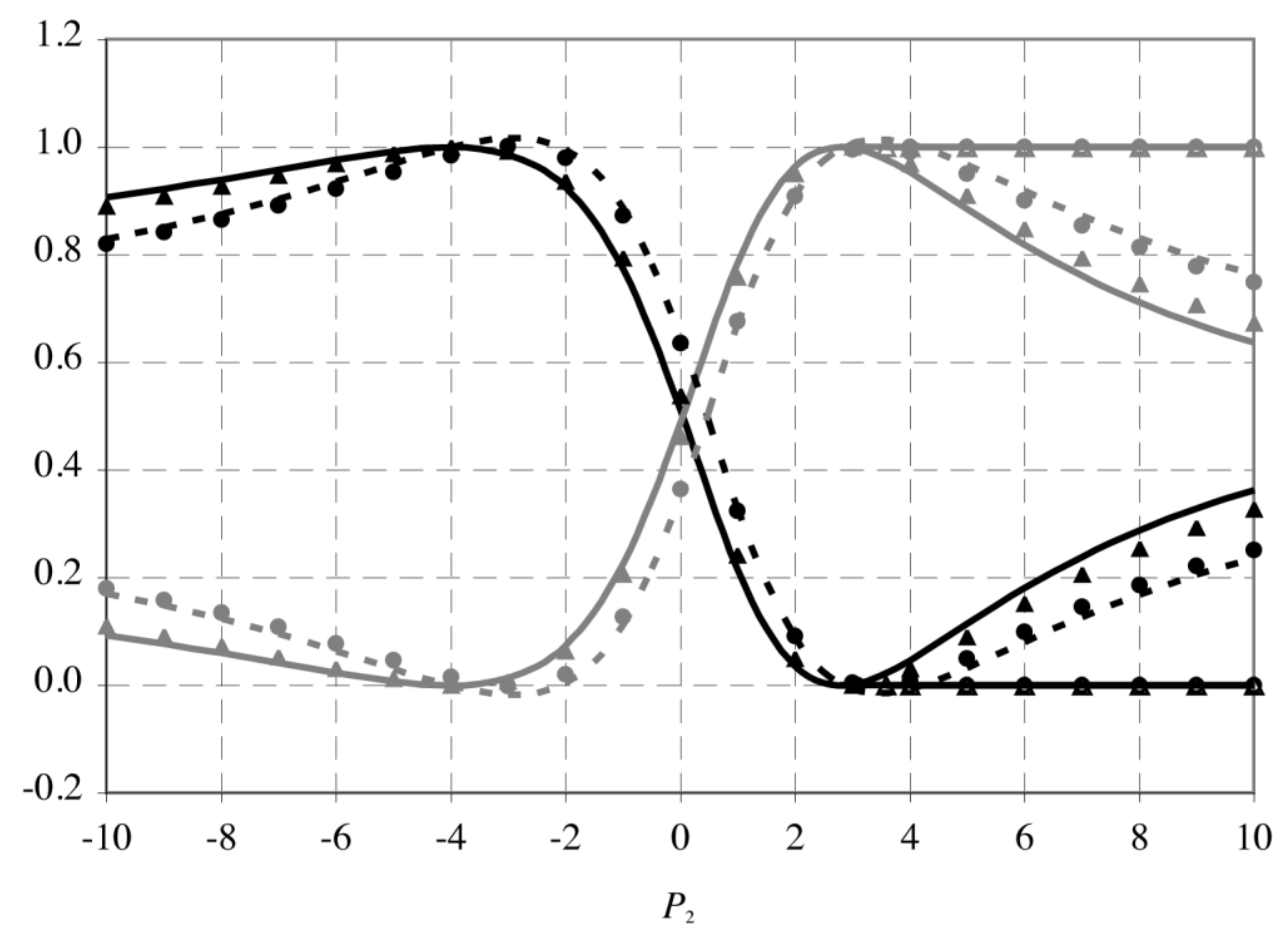

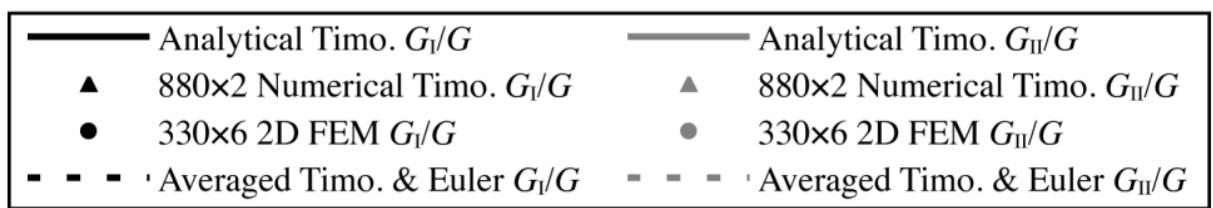

Fig. 11: Comparisons between various theories and FEM simulations with varying $P_{2},\left(M_{1}=0\right.$, $\left.M_{2}=0, N_{1}=0, N_{2}=0, P_{1}=1, G_{x z}=1 / 2.6, \gamma=2\right)$. 


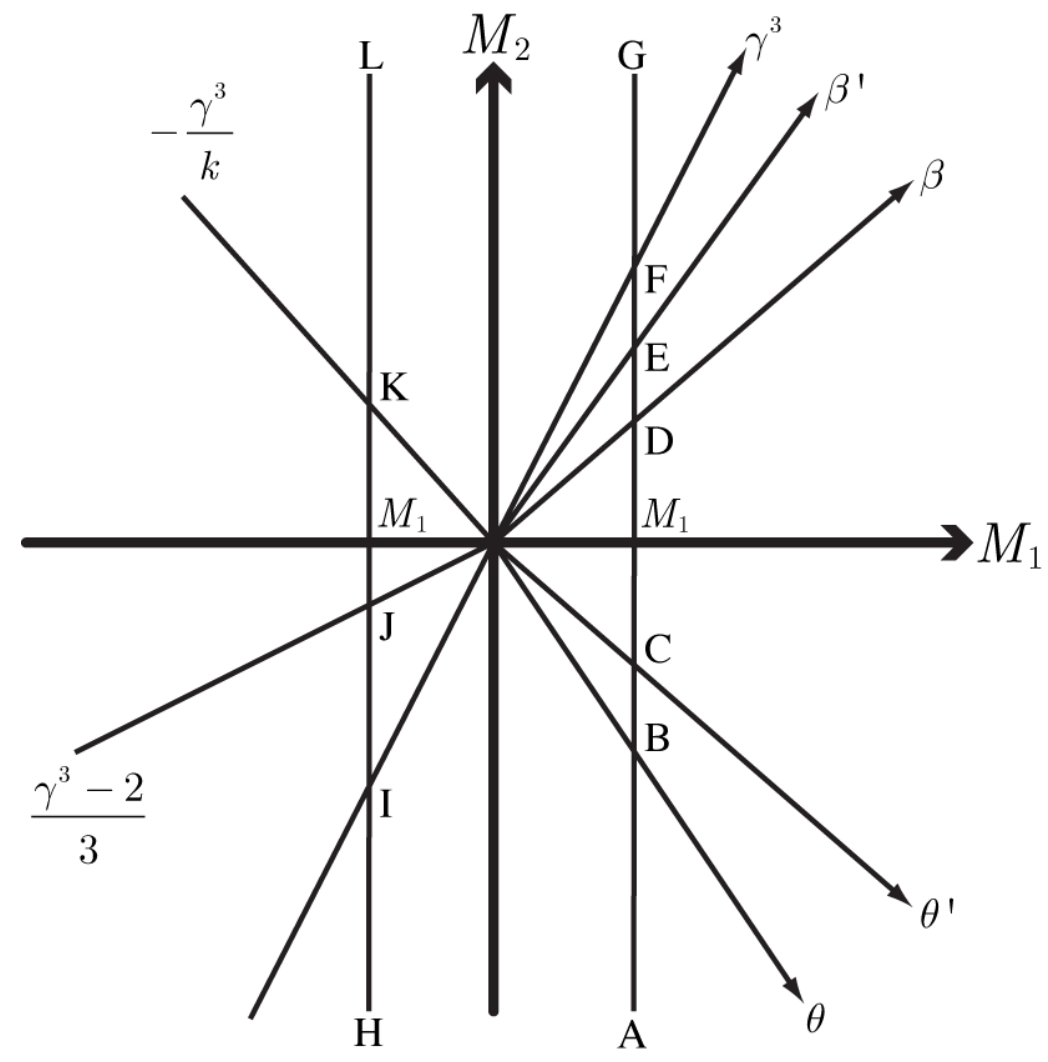

Fig. A1: Fracture mode partition in $\left(M_{1}, M_{2}\right)$ space. 


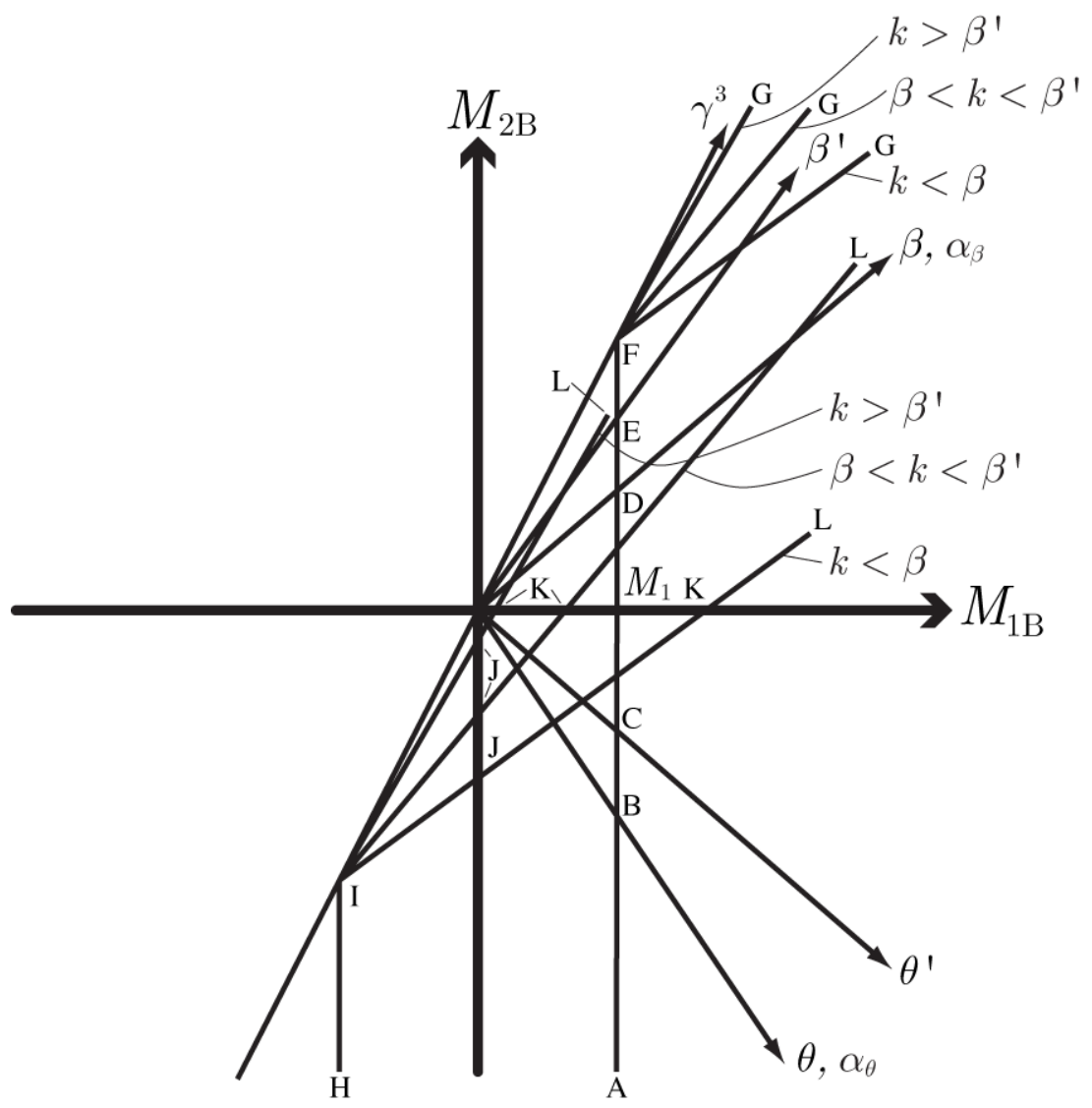

Fig. A2: Fracture mode partition in $\left(M_{1 B}, M_{2 B}\right)$ space. 


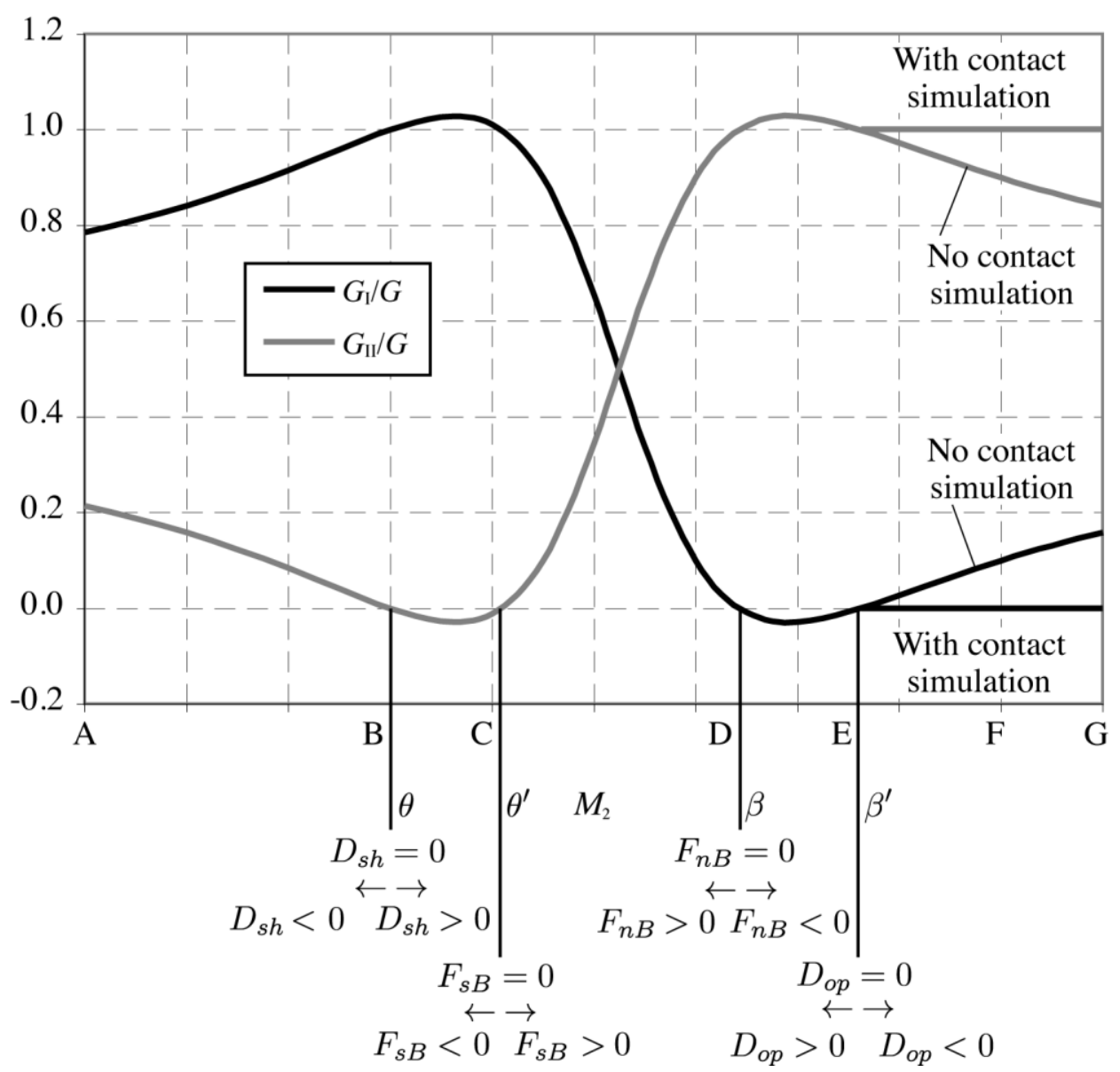

Fig. A3: Variations of $G_{I} / G$ and $G_{I I} / G$ in $\left(M_{1}, M_{2}\right)$ space. 